\title{
KNAPSACK IN HYPERBOLIC GROUPS
}

\author{
MARKUS LOHREY
}

\begin{abstract}
Recently knapsack problems have been generalized from the integers to arbitrary finitely generated groups. The knapsack problem for a finitely generated group $G$ is the following decision problem: given a tuple $\left(g, g_{1}, \ldots, g_{k}\right)$ of elements of $G$, are there natural numbers $n_{1}, \ldots, n_{k} \in \mathbb{N}$ such that $g=g_{1}^{n_{1}} \cdots g_{k}^{n_{k}}$ holds in $G$ ? Myasnikov, Nikolaev, and Ushakov proved that for every (Gromov-)hyperbolic group, the knapsack problem can be solved in polynomial time. In this paper, the precise complexity of the knapsack problem for hyperbolic group is determined: for every hyperbolic group $G$, the knapsack problem belongs to the complexity class LogCFL, and it is LogCFL-complete if $G$ contains a free group of rank two. Moreover, it is shown that for every hyperbolic group $G$ and every tuple $\left(g, g_{1}, \ldots, g_{k}\right)$ of elements of $G$ the set of all $\left(n_{1}, \ldots, n_{k}\right) \in \mathbb{N}^{k}$ such that $g=g_{1}^{n_{1}} \cdots g_{k}^{n_{k}}$ in $G$ is semilinear and a semilinear representation where all integers are of size polynomial in the total geodesic length of the $g, g_{1}, \ldots, g_{k}$ can be computed. Groups with this property are also called knapsack-tame. This enables us to show that knapsack can be solved in LogCFL for every group that belongs to the closure of hyperbolic groups under free products and direct products with $\mathbb{Z}$.
\end{abstract}

\section{INTRODUCTION}

In 22, Myasnikov, Nikolaev, and Ushakov initiated the investigation of discrete optimization problems, which are usually formulated over the integers, for arbitrary (possibly non-commutative) groups. One of these problems is the knapsack problem for a finitely generated group $G$ : The input is a sequence of group elements $g_{1}, \ldots, g_{k}, g \in G$ (specified by finite words over the generators of $G$ ) and it is asked whether there exists a tuple $\left(n_{1}, \ldots, n_{k}\right) \in \mathbb{N}^{k}$ such that $g_{1}^{n_{1}} \cdots g_{k}^{n_{k}}=g$ in $G$. For the particular case $G=\mathbb{Z}$ (where the additive notation $n_{1} \cdot g_{1}+\cdots+n_{k} \cdot g_{k}=g$ is usually preferred) this problem is NP-complete (resp., TC $^{0}$-complete) if the numbers $g_{1}, \ldots, g_{k}, g \in \mathbb{Z}$ are encoded in binary representation [12, 9] (resp., unary notation [2]).

In 22], Myasnikov et al. encode elements of the finitely generated group $G$ by words over the group generators and their inverses, which corresponds to the unary encoding of integers. There is also an encoding of words that corresponds to the binary encoding of integers, so called straight-line programs, and knapsack problems under this encoding have been studied in [18. In this paper, we only consider the case where input words are explicitly represented. Here is a list of known results concerning the knapsack problem:

- Knapsack can be solved in polynomial time for every hyperbolic group 22 . In 4 this result was extended to free products of any finite number of hyperbolic groups and finitely generated abelian groups.

- There are nilpotent groups of class 2 for which knapsack is undecidable. Examples are direct products of sufficiently many copies of the discrete Heisenberg group $H_{3}(\mathbb{Z})[13$, and free nilpotent groups of class 2 and sufficiently high rank 20$]$. 
- Knapsack for $H_{3}(\mathbb{Z})$ is decidable [13. In particular, together with the previous point it follows that decidability of knapsack is not preserved under direct products.

- Knapsack is decidable for every co-context-free group 13, i.e., groups where the set of all words over the generators that do not represent the identity is a context-free language. Lehnert and Schweitzer 15] have shown that the Higman-Thompson groups are co-context-free.

- Knapsack belongs to NP for all virtually special groups (finite extensions of subgroups of graph groups) [19. The class of virtually special groups is very rich. It contains all Coxeter groups, one-relator groups with torsion, fully residually free groups, and fundamental groups of hyperbolic 3-manifolds. For graph groups (also known as right-angled Artin groups) a complete classification of the complexity of knapsack was obtained in [19]: If the underlying graph contains an induced path or cycle on 4 nodes, then knapsack is NP-complete; in all other cases knapsack can be solved in polynomial time (even in LogCFL).

- Decidability of knapsack is preserved under finite extensions, HNN-extensions over finite associated subgroups and amalgamated free products over finite subgroups 18 .

In this paper we further investigate the knapsack problem in hyperbolic groups. The definition of hyperbolic groups requires that all geodesic triangles in the Cayleygraph are $\delta$-slim for a constant $\delta$; see Section 3 for details. The class of hyperbolic groups has several alternative characterizations (e.g., it is the class of finitely generated groups with a linear Dehn function), which gives hyperbolic groups a prominent role in geometric group theory. Moreover, in a certain probabilistic sense, almost all finitely presented groups are hyperbolic $[8,23$. Also from a computational viewpoint, hyperbolic groups have nice properties: it is known that the word problem and the conjugacy problem can be solved in linear time [3, 10. As mentioned above, knapsack can be solved in polynomial time for every hyperbolic group [22]. Our first main result of this paper provides a precise characterization of the complexity of knapsack for hyperbolic groups: for every hyperbolic group, knapsack belongs to LogCFL, which is the class of all problems that are logspace-reducible to a context-free language. LogCFL has several alternative characterizations, see Section 4 for details. The LogCFL upper bound for knapsack in hyperbolic groups improves the polynomial upper bound shown in [22], and also generalizes a result from [16, stating that the word problem for a hyperbolic group is in LogCFL. For hyperbolic groups that contain a copy of a non-abelian free group (such hyperbolic groups are called non-elementary) it follows from [19] that knapsack is LogCFLcomplete. Hyperbolic groups that contain no copy of a non-abelian free group (so called elementary hyperbolic groups) are known to be virtually cyclic, in which case knapsack belongs to nondeterministic logspace (NL), which is contained in LogCFL.

In Section 8 we prove our second main result: for every hyperbolic group $G$ and every tuple $\left(g, g_{1}, \ldots, g_{k}\right)$ of elements of $G$ the set of all $\left(n_{1}, \ldots, n_{k}\right) \in \mathbb{N}^{k}$ such that $g=g_{1}^{n_{1}} \cdots g_{k}^{n_{k}}$ in $G$ is effectively semilinear. In other words: the set of all solutions of a knapsack instance in $G$ is semilinear. Groups with this property are also called knapsack-semilinear. For the special case $G=\mathbb{Z}$ this is well-known (the set of solutions of a linear equation is Presburger definable and hence semilinear). Clearly, knapsack is decidable for every knapsack-semilinear group (due to the effectiveness assumption). In a series of recent papers it turned out that the class of knapsacksemilinear groups is surprisingly rich. It contains all virtually special groups [17. and all co-context-free group [13] and is closed under the following constructions: 
- going to a finitely generated subgroup (this is trivial) and going to a finite group extension [18],

- HNN-extensions over finite associated subgroups and amalgamated free products over finite subgroups [18,

- direct products (in contrast, the class of groups with a decidable knapsack problem is not closed under direct products),

- restricted wreath products $[5$.

Our proof of the knapsack-semilinearity of a hyperbolic group shows an additional quantitative statement: If the group elements $g, g_{1}, \ldots, g_{k}$ are represented by words over the generators and the total length of these words is $N$, then the set $\left\{\left(n_{1}, \ldots, n_{k}\right) \in \mathbb{N}^{k} \mid g=g_{1}^{n_{1}} \cdots g_{k}^{n_{k}}\right.$ in $\left.G\right\}$ has a semilinear representation, where all vectors only contain integers of size at most $p(N)$. Here, $p(x)$ is a fixed polynomial that only depends on $G$. Groups with this property are called knapsack-tame in [19. In [19], it is shown that the class of knapsack-tame groups is closed under free products and direct products with $\mathbb{Z}$. Using this, we can show in Section 9 that knapsack can be solved in LogCFL for every group that belongs to the closure of hyperbolic groups under free products and direct products with $\mathbb{Z}$.

Recently, it was shown that the compressed version of the knapsack problem, where input words are encoded by straight-line programs, is NP-complete for every infinite hyperbolic group [11.

\section{General notations}

We assume that the reader is familiar with basic concepts from group theory and formal languages. The empty word is denoted with $\varepsilon$. For a word $w=a_{1} a_{2} \cdots a_{n}$ let $|w|=n$ be the length of $w$, and for $1 \leq i \leq j \leq n$ let $w[i]=a_{i}, w[i: j]=a_{i} \cdots a_{j}$, $w[: i]=w[1: i]$ and $w[i:]=w[i: n]$. Moreover, let $w[i: j]=\varepsilon$ for $i>j$.

A set of vectors $A \subseteq \mathbb{N}^{k}$ is linear if there exist vectors $v_{0}, \ldots, v_{n} \in \mathbb{N}^{k}$ such that $A=\left\{v_{0}+\lambda_{1} \cdot v_{1}+\cdots+\lambda_{n} \cdot v_{n} \mid \lambda_{1}, \ldots, \lambda_{n} \in \mathbb{N}\right\}$. The tuple of vectors $\left(v_{0}, \ldots, v_{n}\right)$ is a linear represention of $A$. Its magnitude is the largest number appearing in one the vectors $v_{0}, \ldots, v_{n}$. A set $A \subseteq \mathbb{N}^{k}$ is semilinear if it is a finite union of linear sets $A_{1}, \ldots, A_{m}$. A semilinear representation of $A$ is a list of linear representations for the linear sets $A_{1}, \ldots, A_{m}$. Its magnitude is the maximal magnitude of the linear representations for the sets $A_{1}, \ldots, A_{m}$. The magnitude of a semilinear set $A$ is the smallest magnitude among all semilinear representations of $A$.

In the context of knapsack problems, we will consider semilinear sets as sets of mappings $f:\left\{x_{1}, \ldots, x_{k}\right\} \rightarrow \mathbb{N}$ for a finite set of variables $X=\left\{x_{1}, \ldots, x_{k}\right\}$. Such a mapping $f$ can be identified with the vector $\left(f\left(x_{1}\right), \ldots, f\left(x_{k}\right)\right)$. This allows to use all vector operations (e.g. addition and scalar multiplication) on the set $\mathbb{N}^{X}$ of all mappings from $X$ to $\mathbb{N}$. The pointwise product $f \cdot g$ of two mappings $f, g \in \mathbb{N}^{X}$ is defined by $(f \cdot g)(x)=f(x) \cdot g(x)$ for all $x \in X$. Moreover, for mappings $f \in \mathbb{N}^{X}$, $g \in \mathbb{N}^{Y}$ with $X \cap Y=\emptyset$ we define $f \oplus g: X \cup Y \rightarrow \mathbb{N}$ by $(f \oplus g)(x)=f(x)$ for $x \in X$ and $(f \oplus g)(y)=g(y)$ for $y \in Y$. All operations on $\mathbb{N}^{X}$ will be extended to subsets of $\mathbb{N}^{X}$ in the standard pointwise way.

It is well-known that the semilinear subsets of $\mathbb{N}^{k}$ are exactly the sets definable in Presburger arithmetic. These are those sets that can be defined with a first-order formula $\varphi\left(x_{1}, \ldots, x_{k}\right)$ over the structure $(\mathbb{N}, 0,+, \leq)$ [7. Moreover, the transformations between such a first-order formula and an equivalent semilinear representation are effective. In particular, the semilinear sets are effectively closed under Boolean operations. 


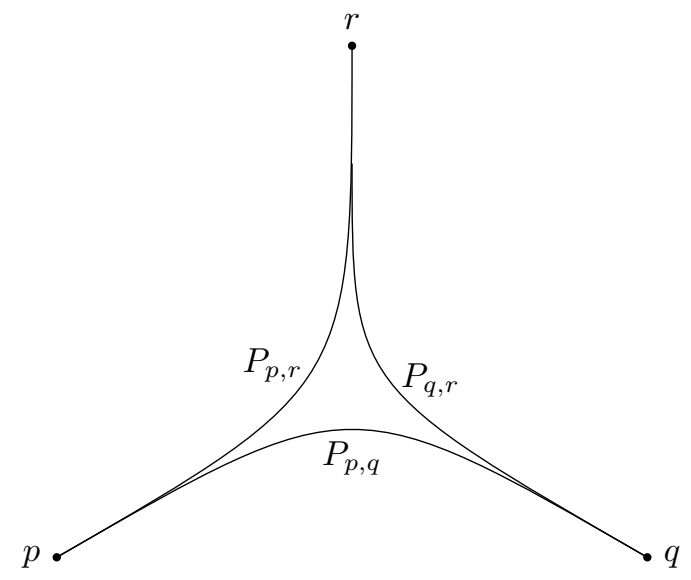

FiguRE 1. The shape of a geodesic triangle in a hyperbolic group

\section{HyPERBOLIC GROUPS}

Let $G$ be a finitely generated group with the finite symmetric generating set $\Sigma$, i.e., $a \in \Sigma$ implies that $a^{-1} \in \Sigma$. The Cayley-graph of $G$ (with respect to $\Sigma$ ) is the undirected graph $\Gamma=\Gamma(G)$ with node set $G$ and all edges $(g, g a)$ for $g \in G$ and $a \in \Sigma$. We view $\Gamma$ as a geodesic metric space, where every edge $(g, g a)$ is identified with a unit-length interval. It is convenient to label the directed edge from $g$ to $g a$ with the generator $a$. The distance between two points $p, q$ is denoted with $d_{\Gamma}(p, q)$. For $g \in G$ let $|g|=d_{\Gamma}(1, g)$. For $r \geq 0$, let $\mathcal{B}_{r}(1)=\left\{g \in G \mid d_{\Gamma}(1, g) \leq r\right\}$.

Paths can be defined in a very general way for metric spaces, but we only need paths that are induced by words over $\Sigma$. Given a word $w \in \Sigma^{*}$ of length $n$, one obtains a unique path $P[w]:[0, n] \rightarrow \Gamma$, which is a continuous mapping from the real interval $[0, n]$ to $\Gamma$. It maps the subinterval $[i, i+1] \subseteq[0, n]$ isometrically onto the edge $\left(g_{i}, g_{i+1}\right)$ of $\Gamma$, where $g_{i}$ (resp., $\left.g_{i+1}\right)$ is the group element represented by the word $w[: i]$ (resp., $w[: i+1]$ ). The path $P[w]$ starts in $1=g_{0}$ and ends in $g_{n}$ (the group element represented by $w$ ). We also say that $P[w]$ is the unique path that starts in 1 and is labelled with the word $w$. More generally, for $g \in G$ we denote with $g \cdot P[w]$ the path that starts in $g$ and is labelled with $w$. When writing $u \cdot P[w]$ for a word $u \in \Sigma^{*}$, we mean the path $g \cdot P[w]$, where $g$ is the group element represented by $u$. A path $P:[0, n] \rightarrow \Gamma$ of the above form is geodesic if $d_{\Gamma}(P(0), P(n))=n$; it is a $(\lambda, \epsilon)$-quasigeodesic if for all points $p=P(a)$ and $q=P(b)$ we have $|a-b| \leq \lambda \cdot d_{\Gamma}(p, q)+\varepsilon$; and it is $\zeta$-local $(\lambda, \epsilon)$-quasigeodesic if for all points $p=P(a)$ and $q=P(b)$ with $|a-b| \leq \zeta$ we have $|a-b| \leq \lambda \cdot d_{\Gamma}(p, q)+\varepsilon$.

A word $w \in \Sigma^{*}$ is geodesic if the path $P[w]$ is geodesic, which means that there is no shorter word representing the same group element from $G$. Similarly, we define the notion of ( $\zeta$-local) $(\lambda, \epsilon)$-quasigeodesic words. A word $w \in \Sigma^{*}$ is shortlex reduced if it is the length-lexicographically smallest word that represents the same group element as $w$. For this, we have to fix an arbitrary linear order on $\Sigma$. Note that if $u=x y$ is shortlex reduced then $x$ and $y$ are shortlex reduced too. For a word $u \in \Sigma^{*}$ we denote with $\operatorname{shlex}(u)$ the unique shortlex reduced word that represents the same group element as $u$.

A geodesic triangle consists of three points $p, q, r \in G$ and geodesic paths $P_{1}=$ $P_{p, q}, P_{2}=P_{p, r}, P_{3}=P_{q, r}$ (the three sides of the triangle), where $P_{x, y}$ is a geodesic path from $x$ to $y$. We call a geodesic triangle $\delta$-slim for $\delta \geq 0$, if for all $i \in\{1,2,3\}$, every point on $P_{i}$ has distance at most $\delta$ from a point on $P_{j} \cup P_{k}$, where $\{j, k\}=$ $\{1,2,3\} \backslash\{i\}$. The group $G$ is called $\delta$-hyperbolic, if every geodesic triangle is $\delta$-slim. 


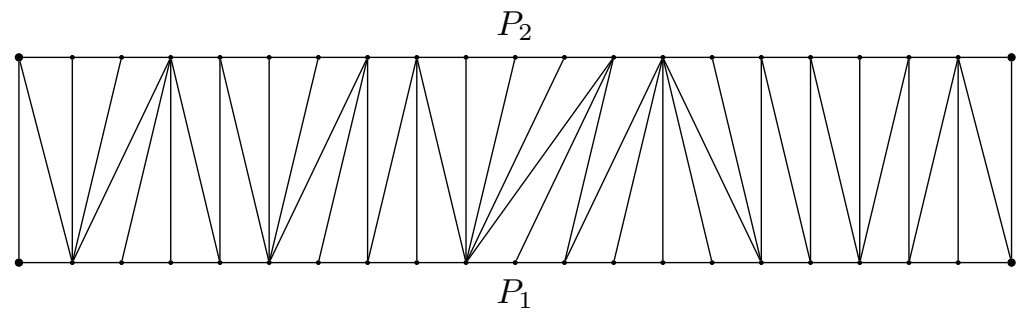

FiguRE 2. Paths that asynchronously $K$-fellow travel

Finally, $G$ is hyperbolic, if it is $\delta$-hyperbolic for some $\delta \geq 0$. Figure 1 shows the shape of a geodesic triangle in a hyperbolic group. Finitely generated free groups are for instance 0 -hyperbolic. The property of being hyperbolic is independent of the chosen generating set $\Sigma$. The word problem for every hyperbolic group can be decided in real time 10 .

Let us fix a $\delta$-hyperbolic group $G$ with the finite symmetric generating set $\Sigma$ for the rest of the section, and let $\Gamma$ be the corresponding geodesic metric space. We will apply a couple of well-known results for hyperbolic groups.

Lemma 3.1 (c.f. [6, 8.21]). Let $g \in G$ be of infinite order and let $n \geq 0$. Let $u$ be a geodesic word representing $g$. Then the word $u^{n}$ is $(\lambda, \epsilon)$-quasigeodesic, where $\lambda=N|g|, \epsilon=2 N^{2}|g|^{2}+2 N|g|$ and $N=\left|\mathcal{B}_{2 \delta}(1)\right|$.

Consider two paths $P_{1}:\left[0, n_{1}\right] \rightarrow \Gamma, P_{2}:\left[0, n_{2}\right] \rightarrow \Gamma$ and let $K$ be a positive real number. We say that $P_{1}$ and $P_{2}$ asynchronously $K$-fellow travel if there exist two continuous non-decreasing mappings $\varphi_{1}:[0,1] \rightarrow\left[0, n_{1}\right]$ and $\varphi_{2}:[0,1] \rightarrow\left[0, n_{2}\right]$ such that $\varphi_{1}(0)=\varphi_{2}(0)=0, \varphi_{1}(1)=n_{1}, \varphi_{2}(1)=n_{2}$ and for all $0 \leq t \leq 1$, $d_{\Gamma}\left(P_{1}\left(\varphi_{1}(t)\right), P_{2}\left(\varphi_{2}(t)\right)\right) \leq K$. Intuitively, this means that one can travel along the paths $P_{1}$ and $P_{2}$ asynchronously with variable speeds such that at any time instant the current points have distance at most $K$. By slightly increasing $K$ one obtains a ladder graph of the form shown in Figure 2. where the edges connecting the horizontal $P_{1}$ - and $P_{2}$-labelled paths represent paths of length at most $K$ that connect elements from $G$.

Lemma 3.2 (c.f. 21]). Let $P_{1}$ and $P_{2}$ be $(\lambda, \epsilon)$-quasigeodesic paths in $\Gamma_{G}$ and assume that $P_{i}$ starts in $g_{i}$ and ends in $h_{i}$. Assume that $d_{\Gamma}\left(g_{1}, g_{2}\right), d_{\Gamma}\left(h_{1}, h_{2}\right) \leq h$. Then there exists a computable bound $K=K(\delta, \lambda, \epsilon, h) \geq h$ such that $P_{1}$ and $P_{2}$ asynchronously $K$-fellow travel.

Finally we need the following lemma for splitting quasigeodesic rectangles:

Lemma 3.3. Fix constants $\lambda, \epsilon$ and let $\kappa=K(\delta, \lambda, \epsilon, 0)$ be taken from Lemma 3.2. Let $v_{1}, v_{2} \in \Sigma^{*}$ be geodesic words and $u_{1}, u_{2} \in \Sigma^{*}(\lambda, \epsilon)$-quasigeodesic words such that $v_{1} u_{1}=u_{2} v_{2}$ in $G$. Consider a factorization $u_{1}=x_{1} y_{1}$ with $\left|x_{1}\right| \geq \lambda\left(\left|v_{1}\right|+2 \delta+\right.$ $\kappa)+\epsilon$ and $\left|y_{1}\right| \geq \lambda\left(\left|v_{2}\right|+2 \delta+\kappa\right)+\epsilon$ Then there exists a factorization $u_{2}=x_{2} y_{2}$ and $c \in \mathcal{B}_{2 \delta+2 \kappa}(1)$ such that $v_{1} x_{1}=x_{2} c$ and $c y_{1}=y_{2} v_{2}$ in $G$.

Proof. The construction is shown in Figure 3.3. Let $t_{1}, t_{2}, x_{1}^{\prime}, y_{1}^{\prime}$ be geodesic words with $t_{1}=u_{1}, t_{2}=u_{2}, x_{1}=x_{1}^{\prime}$ and $y_{1}=y_{1}^{\prime}$ in $G$. Since $u_{1}$ is $(\lambda, \epsilon)$-quasigeodesic, we get $\left|x_{1}^{\prime}\right| \geq\left(\left|x_{1}\right|-\epsilon\right) / \lambda \geq\left|v_{1}\right|+2 \delta+\kappa$ and $\left|y_{1}^{\prime}\right| \geq\left(\left|y_{1}\right|-\epsilon\right) / \lambda \geq\left|v_{2}\right|+2 \delta+\kappa$. By Lemma 3.2 the paths $P\left[t_{1}\right]$ and $P\left[u_{1}\right]$ asynchronously $\kappa$-fellow travel. Hence, there exists a factorization $t_{1}=r_{1} s_{1}$ and $c_{1} \in \mathcal{B}_{\kappa}(1)$ such that $r_{1} c_{1}=x_{1}=x_{1}^{\prime}$ and $c_{1} y_{1}^{\prime}=c_{1} y_{1}=s_{1}$ in $G$. This implies $\left|r_{1}\right| \geq\left|x_{1}^{\prime}\right|-\kappa \geq\left|v_{1}\right|+2 \delta$ and $\left|s_{1}\right| \geq\left|y_{1}^{\prime}\right|-\kappa \geq$ $\left|v_{2}\right|+2 \delta$. Consider the geodesic rectangle with the paths $Q_{1}=P\left[v_{1}\right], P_{1}=v_{1} \cdot P\left[t_{1}\right]$, $P_{2}=P\left[t_{2}\right]$, and $Q_{2}=u_{2} \cdot P\left[v_{2}\right]$. Since geodesic rectangles are $2 \delta$-slim, there exists 


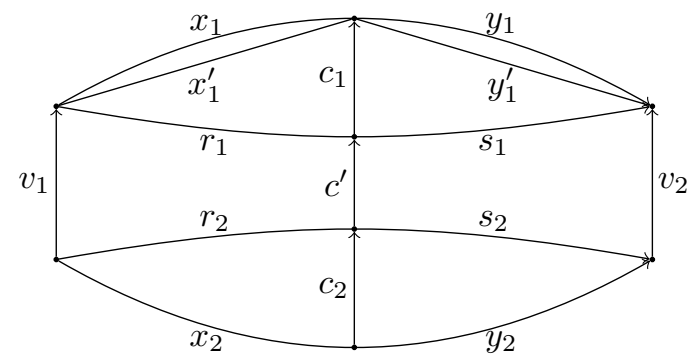

FIGURE 3. Splitting a quasigeodesic rectangle according to Lemma 3.3

a point $p_{2} \in P_{2} \cup Q_{1} \cup Q_{2}$ that has distance at most $2 \delta$ from $p_{1}=P_{1}\left(\left|r_{1}\right|\right)$. By the triangle inequality we must have $p_{2} \in P_{2}$. This yields a factorization $t_{2}=r_{2} s_{2}$ (where $p_{2}=P_{2}\left(\left|r_{2}\right|\right)$ ) and $c^{\prime} \in \mathcal{B}_{2 \delta}(1)$ such that $v_{1} r_{1}=r_{2} c^{\prime}$ and $c^{\prime} s_{1}=s_{2} v_{2}$ in $G$. Finally, since $P\left[t_{2}\right]$ and $P\left[u_{2}\right]$ asynchronously $\kappa$-fellow travel, we obtain a factorization $u_{2}=x_{2} y_{2}$ and $c_{2} \in \mathcal{B}_{\kappa}(1)$ such that $x_{2} c_{2}=r_{2}$ and $c_{2} s_{2}=y_{2}$ in $G$. Let $c=c_{2} c^{\prime} c_{1} \in \mathcal{B}_{2 \delta+2 \kappa}(1)$. We get $x_{2} c=x_{2} c_{2} c^{\prime} c_{1}=r_{2} c^{\prime} c_{1}=v_{1} r_{1} c_{1}=v_{1} x_{1}$ and $c y_{1}=c_{2} c^{\prime} c_{1} y_{1}=c_{2} c^{\prime} s_{1}=c_{2} s_{2} v_{2}=y_{2} v_{2}$.

\section{The COMPlEXity Class LogCFL}

The complexity class LogCFL consists of all computational problems that are logspace reducible to a context-free language. The class LogCFL is included in the parallel complexity class $\mathrm{NC}^{2}$ and has several alternative characterizations (see e.g. [24, 26]):

- logspace bounded alternating Turing-machines with polynomial tree size,

- semi-unbounded Boolean circuits of polynomial size and logarithmic depth, and

- logspace bounded auxiliary pushdown automata with polynomial running time.

For our purposes, the last characterization is most suitable. An AuxPDA (for auxiliary pushdown automaton) is a nondeterministic pushdown automaton with a two-way input tape and an additional work tape. Here we only consider AuxPDAs with the following two restrictions:

- The length of the work tape is restricted to $O(\log n)$ for an input of length $n$ (logspace bounded).

- There is a polynomial $p(n)$, such that every computation path of the AuxPDA on an input of length $n$ has length at most $p(n)$ (polynomially time bounded).

Whenever we speak of an AuxPDA in the following, we implicitly assume that the AuxPDA is logspace bounded and polynomially time bounded. The class of languages that are accepted by AuxPDAs is exactly LogCFL [24]. A one-way AuxPDA is an AuxPDA that never moves the input head to the left. Hence, in every step, the input head either does not move, or moves to the right.

For a finitely generated group $G$ with the symmetric generating set $\Sigma$ we define the word problem for $G$ (with respect to $\Sigma$ ) as the set of all words $w \in \Sigma^{*}$ such that $w=1$ in $G$. Let us say that a finitely generated group $G$ belongs to the class OW-AuxPDA if the word problem for $G$ is recognized by a one-way AuxPDA. It is easy to see that the latter property is independent of the generating set of $G$ (this 
holds, since the class of languages recognized by one-way AuxPDAs is closed under inverse homomorphisms).

Theorem 4.1. Every hyperbolic group belongs to the class $O W-A u x P D A$.

Proof. Let $G$ be a hyperbolic group. In [16] it is shown that the word problem for $G$ is a growing context-sensitive language, i.e., it can be generated by a grammar where all productions are strictly length-increasing (except for the start production $S \rightarrow \varepsilon$ ). In [1] it was shown that every growing context-sensitive language can be recognized by a one-way AuxPDA in logarithmic space and polynomial time. The result follows.

Theorem 4.2. If the groups $G$ and $H$ belong to $O W$-AuxPDA then also $G * H$ and $G \times \mathbb{Z}$ belong to $O W-A u x P D A$.

Proof. The proof is essentially the same as in [19, Lemma 4.8], but is presented for completeness. Let us first consider the group $G \times \mathbb{Z}$. Let $\mathcal{P}(G)$ be a one-way AuxPDA for the word problem of $G$. The one-way AuxPDA $\mathcal{P}(G \times \mathbb{Z})$ for the word problem of $G$ simulates $\mathcal{P}(G)$ on the generators of $G$. Moreover, it stores the current value of the $\mathbb{Z}$-component in binary notation on the work tape. If the input word has length $n$, then $O(\log n)$ bits are sufficient for this. At the end, $\mathcal{P}(G \times \mathbb{Z})$ accepts if and only if $\mathcal{P}(G)$ accepts and the $\mathbb{Z}$-component on the work tape is zero.

Next, we consider the group $G * H$. We have one-way AuxPDAs $\mathcal{P}(G)$ and $\mathcal{P}(H)$ for the word problems of $G$ and $H$, respectively. We can assume that $\mathcal{P}(G)$ (resp., $\mathcal{P}(H)$ ) accepts an input word $w$ if after reading $w$ the stack is empty and $\mathcal{P}(G)$ (resp., $\mathcal{P}(H)$ ) is in the unique final state $q_{G}$ (resp., $q_{H}$ ). This can be achieved by doing $\varepsilon$-transitions at the end of the computation. In the following, we call $q_{G}$ (resp., $q_{H}$ ) the 1 -state of $\mathcal{P}(G)$ (resp., $\mathcal{P}(H)$ ).

Let $\Sigma$ (resp., $\Gamma$ ) be the input alphabet of $\mathcal{P}(G)$ (resp., $\mathcal{P}(H)$ ), which is a symmetric generating set for $G$ (resp., $H$ ). We assume that $\Sigma \cap \Gamma=\emptyset$. Consider now an input word $w \in(\Sigma \cup \Gamma)^{*}$. Let us assume that $w=u_{1} v_{1} u_{2} v_{2} \cdots u_{k} v_{k}$ with $u_{i} \in \Sigma^{+}$ and $v_{i} \in \Gamma^{+}$(other cases can be treated analogously). The AuxPDA $\mathcal{P}(G * H)$ starts with empty stack and simulates the AuxPDA $\mathcal{P}(G)$ on the prefix $u_{1}$. If it turns out that $u_{1}=1$ in $G$ (which means that $\mathcal{P}(G)$ is in its 1-state and the stack is empty) then the AuxPDA $\mathcal{P}(G * H)$ continues with simulating $\mathcal{P}(H)$ on $v_{1}$. On the other hand, if $u_{1} \neq 1$ in $G$, then $\mathcal{P}(G * H)$ pushes the state together with the work tape content of $\mathcal{P}(G)$ reached after reading $u_{1}$ on the stack (on top of the final stack content of $\mathcal{P}(G)$ ). This allows $\mathcal{P}(G * H)$ to resume the computation of $\mathcal{P}(G)$ later. Then $\mathcal{P}(G * H)$ continues with simulating $\mathcal{P}(H)$ on $v_{1}$.

The computation of $\mathcal{P}(G * H)$ will continue in this way. More precisely, if after reading $u_{i}$ (resp. $v_{i}$ with $i<k$ ) the AuxPDA $\mathcal{P}(G)$ (resp. $\mathcal{P}(H)$ ) is in its 1-state then either

(i) the stack is empty or

(ii) the top part of the stack is of the form $s q t$ ( $t$ is the top), where $s$ is a stack content of $\mathcal{P}(H)$ (resp. $\mathcal{P}(G)), q$ is a state of $\mathcal{P}(H)$ (resp. $\mathcal{P}(G)$ ) and $t$ is a work tape content of $\mathcal{P}(H)$ (resp. $\mathcal{P}(G)$ ).

In case (i), $\mathcal{P}(G * H)$ continues with the simulation of $\mathcal{P}(H)$ (resp. $\mathcal{P}(G)$ ) on the word $v_{i}$ (resp. $u_{i+1}$ ) in the initial configuration. In case (ii), $\mathcal{P}(G * H)$ continues with the simulation of $\mathcal{P}(H)$ (resp. $\mathcal{P}(G)$ ) on the word $v_{i}$ (resp. $u_{i+1}$ ), where the simulation is started with stack content $s$, state $q$, and work tape content $t$. On the other hand, if after reading $u_{i}$ (resp. $v_{i}$ with $i<k$ ) the $\operatorname{AuxPDA} \mathcal{P}(G)$ (resp. $\mathcal{P}(H))$ is not in its 1 -state then $\mathcal{P}(G * H)$ pushes on the stack the state and work tape content of $\mathcal{P}(G)$ reached after its simulation on $u_{i}$. This concludes the description of the AuxPDA $\mathcal{P}(G * H)$. It is a one-way AuxPDA that accepts the word problem of $G * H$. 


\section{KNAPSACK PROBLEMS}

Let $G$ be a finitely generated group with the finite symmetric generating set $\Sigma$. Moreover, let $X$ be a set of formal variables that take values from $\mathbb{N}$. For a subset $U \subseteq X$, we use $\mathbb{N}^{U}$ to denote the set of maps $\nu: U \rightarrow \mathbb{N}$, which we call valuations. An exponent expression over $G$ is a formal expression of the form $E=u_{1}^{x_{1}} v_{1} u_{2}^{x_{2}} v_{2} \cdots u_{k}^{x_{k}} v_{k}$ with $k \geq 1$ and words $u_{i}, v_{i} \in \Sigma^{*}$. Here, the variables do not have to be pairwise distinct. If every variable in an exponent expression occurs at most once, it is called a knapsack expression. Let $X_{E}=\left\{x_{1}, \ldots, x_{k}\right\}$ be the set of variables that occur in $E$. For a valuation $\nu \in \mathbb{N}^{U}$ such that $X_{E} \subseteq U$ (in which case we also say that $\nu$ is a valuation for $E$ ), we define $\nu(E)=u_{1}^{\nu\left(x_{1}\right)} v_{1} u_{2}^{\nu\left(x_{2}\right)} v_{2} \cdots u_{k}^{\nu\left(x_{k}\right)} v_{k} \in \Sigma^{*}$. We say that $\nu$ is a solution of the equation $E=1$ if $\nu(E)$ evaluates to the identity element 1 of $G$. With $\operatorname{sol}(E)$ we denote the set of all solutions $\nu \in \mathbb{N}^{X_{E}}$ of $E$. We can view $\operatorname{sol}(E)$ as a subset of $\mathbb{N}^{k}$. The length of $E$ is defined as $|E|=\sum_{i=1}^{k}\left|u_{i}\right|+\left|v_{i}\right|$, whereas $k$ is its depth. We define solvability of exponent equations over $G$ as the following decision problem:

Input: A finite list of exponent expressions $E_{1}, \ldots, E_{n}$ over $G$.

Question: Is $\bigcap_{i=1}^{n}$ sol $\left(E_{i}\right)$ non-empty?

The knapsack problem for $G$ is the following decision problem:

Input: A single knapsack expression $E$ over $G$.

Question: Is sol $(E)$ non-empty?

It is easy to observe that the concrete choice of the generating set $\Sigma$ has no influence on the decidability and complexity status of these problems. Later, we will also allow exponent expressions of the form $v_{0} u_{1}^{x_{1}} v_{1} u_{2}^{x_{2}} v_{2} \cdots u_{k}^{x_{k}} v_{k}$, which do not start with a power $u_{1}^{x_{1}}$. Such an exponent expression can be replaced by $u_{1}^{x_{1}} v_{1} u_{2}^{x_{2}} v_{2} \cdots u_{k}^{x_{k}} v_{k} v_{0}$ without changing the set of solutions.

The group $G$ is called knapsack-semilinear if for every knapsack expression $E$ over $G$, the set $\operatorname{sol}(E)$ is a semilinear set of vectors and a semilinear representation can be effectively computed from $E$. Since the emptiness of the intersection of finitely many semilinear sets is decidable, solvability of exponent equations is decidable for every knapsack-semilinear group. As mentioned in the introduction, the class of knapsack-semilinear groups is very rich. An example of a group $G$, where knapsack is decidable but solvability of exponent equations is undecidable is the Heisenberg group $H_{3}(\mathbb{Z})$ (which consists of all upper triangular $(3 \times 3)$-matrices over the integers, where all diagonal entries are 1$)$, see $\left[13\right.$. In particular, $H_{3}(\mathbb{Z})$ is not knapsacksemilinear.

The group $G$ is called polynomially knapsack-bounded if there is a fixed polynomial $p(n)$ such that for a given a knapsack expression $E$ over $G$, one has $\operatorname{sol}(E) \neq \emptyset$ if and only if there exists $\nu \in \operatorname{sol}(E)$ with $\nu(x) \leq p(|E|)$ for all variables $x$ in $E$.

The group $G$ is called knapsack-tame if there is a fixed polynomial $p(n)$ such that for a given a knapsack expression $E$ over $G$ one can compute a semilinear representation for $\operatorname{sol}(E)$ of magnitude at most $p(|E|)$. Thus, every knapsack-tame group is knapsack-semilinear as well as polynomially knapsack-bounded. The following result was shown in 19]:

Proposition 5.1 ([19, Proposition 4.11 and 4.17]). If $G$ and $H$ are knapsack-tame groups then also the free product $G * H$ and the direct product $G \times \mathbb{Z}$ are knapsacktame.

\section{Membership FOr ACYCliC AUtomata}

An acyclic NFA is a nondeterministic finite automaton $\mathcal{A}=\left(Q, \Sigma, \Delta, q_{0}, F\right)(Q$ is a finite set of states, $\Sigma$ is the input alphabet, $\Delta \subseteq Q \times \Sigma^{*} \times Q$ is the set of transition 
triples, $q_{0} \in Q$ is the initial state, and $F \subseteq Q$ is the set of final states) such that the relation $\left\{(p, q) \in Q \times Q \mid \exists w \in \Sigma^{*}:(p, w, q) \in \Delta\right\}$ is acyclic. Note that we allow transitions labelled with words, which will be convenient in the following.

Let $G$ be a finitely generated group with the finite symmetric generating set $\Sigma$. The membership problem for acyclic NFAs over $G$ is the following computational problem:

Input: an acyclic NFA $\mathcal{A}$ with input alphabet $\Sigma$.

Question: does $\mathcal{A}$ accept a word $w \in \Sigma^{*}$ such that $w=1$ in $G$ ?

Again, the concrete choice of the generating set $\Sigma$ has no influence on the decidability and complexity status of this problem.

Theorem 6.1. If the group $G$ belongs to the class $O W$-AuxPDA, then membership for acyclic NFAs over $G$ belongs to LogCFL.

Proof. Let $\mathcal{P}$ be a one-way AuxPDA for the word problem of $G$. An AuxPDA for the membership problem for acyclic NFAs over $G$ guesses a path in the acyclic input NFA $\mathcal{A}$ and thereby simulates the AuxPDA $\mathcal{P}$ on the word spelled by the guessed path. If the final state of the input NFA $\mathcal{A}$ is reached and the AuxPDA $\mathcal{P}$ accepts at the same time, then the overall AuxPDA accepts. It is important that the AuxPDA $\mathcal{P}$ works one-way since the guessed path in $\mathcal{A}$ cannot be stored in logspace. This implies that the AuxPDA cannot re-access the input symbols that have already been processed. Also note that the AuxPDA is logspace bounded and polynomially time bounded since $\mathcal{A}$ is acyclic.

Theorem 6.2. Let $G$ be a polynomially knapsack-bounded group. Then there is a logspace reduction from the knapsack problem for $G$ to membership for acyclic NFAs over $G$.

Proof. Let $G$ be a polynomially knapsack-bounded group with the symmetric generating set $\Sigma$. We present a logspace reduction from knapsack for $G$ to the membership problem for acyclic NFAs. Consider a knapsack expression $E=u_{1}^{x_{1}} v_{1} u_{2}^{x_{2}} v_{2} \cdots u_{k}^{x_{k}} v_{k}$ over $G$. Since $G$ is polynomially knapsack-bounded, there exists a polynomial $p(x)$ such that $\operatorname{sol}(E) \neq \emptyset$ if and only if there exists a solution $\nu \in \operatorname{sol}(E)$ such that $\nu\left(x_{i}\right) \leq p(|E|)$ for all $1 \leq i \leq k$. We now construct an NFA $\mathcal{A}$ as follows: It has the state set $Q=[1, k+1] \times[0, p(n)]$ and the following transitions. For each $i \in[1, k]$ and $j \in[0, p(n)-1]$, there are two transitions from $(i, j)$ to $(i, j+1)$; one labeled by $u_{i}$ and one labeled by $\varepsilon$. Furthermore, there is a transition from $(i, p(n))$ to $(i+1,0)$ labeled $v_{i}$ for each $i \in[1, k]$. The initial state is $(1,0)$ and the unique final state is $(k+1,0)$.

It is clear that $\mathcal{A}$ accepts a word that represents 1 if and only if $\operatorname{sol}(E) \neq \emptyset$. Finally, the NFA can be clearly computed in logarithmic space from $E$.

\section{COMPLEXITY OF KNAPSACK IN HYPERBOLIC GROUPS}

In this section we consider the complexity of the knapsack problem for a hyperbolic group. In 22 it was shown that for every hyperbolic group, knapsack can be solved in polynomial time. Here, we improve the complexity to LogCFL. We need one more result from [22]:

Theorem 7.1 (c.f. 22]). Every hyperbolic group is polynomially knapsack-bounded.

This result is also a direct corollary of Theorem 8.1 from the next section, stating that every hyperbolic group is knapsack-tame.

We can now easily derive the following two results:

Corollary 7.2. Membership for acyclic NFAs over a hyperbolic group belongs to LogCFL. 
Proof. This follows from Theorem 4.1 and 6.1

Corollary 7.3. For every hyperbolic groups $G$, knapsack can be solved in LogCFL. Moreover, if $G$ contains a copy of $F_{2}$ (the free group of rank 2) then knapsack for $G$ is LogCFL-complete.

Proof. The first statement follows from Theorems 6.2 and 7.1 and Corollary 7.2 The second statement follows from [19, Proposition 4.26], where it was shown that knapsack for $F_{2}$ is LogCFL-complete.

\section{HyPERBoliC GROUPS ARE KNAPSACK-SEMILINEAR}

In this section, we prove the following strengthening of Theorem 7.1 ;

Theorem 8.1. Every hyperbolic group is knapsack-tame.

Let us remark that the total number of vectors in a semilinear representation can be exponential, even for the simplest case $G=\mathbb{Z}$. Take the (additively written) knapsack expression $E=x_{1}+x_{2}+\cdots+x_{n}-n$. Then $\operatorname{sol}(E)$ is finite and consists of $\left(\begin{array}{c}2 n-1 \\ n\end{array}\right) \geq 2^{n}$ vectors.

Let us fix a $\delta$-hyperbolic group $G$ for the rest of Section 8 and let $\Sigma$ be a finite symmetric generating set for $G$.

8.1. Knapsack expressions of depth two. We first consider knapsack expressions of depth 2 where all powers are quasigeodesic. It is well known that the semilinear sets are exactly the Parikh images of the regular languages. We need a quantitative version of this result that was independently discovered by Kopczynski and Lin:

Theorem 8.2 (c.f. [25, Theorem 4.1], see also [14]). Let $k$ be a fixed constant. Given an NFA $\mathcal{A}$ over an alphabet of size $k$ with $n$ states, one can compute in polynomial time a semilinear representation of the Parikh image of $L(\mathcal{A})$. Moreover, all numbers appearing in the semilinear representation are polynomially bounded in $n$ (in other words: one can compute the semilinear representation with unary encoded numbers).

Lemma 8.3. Let $\lambda$ and $\epsilon$ be fixed constants. For all geodesic words $u_{1}, v_{1}, u_{2}, v_{2} \in$ $\Sigma^{*}$ such that $u_{1} \neq \varepsilon \neq u_{2}$ and $u_{1}^{n}, u_{2}^{n}$ are $(\lambda, \epsilon)$-quasigeodesic for all $n \geq 0$, the set $\left\{\left(x_{1}, x_{2}\right) \in \mathbb{N} \times \mathbb{N} \mid v_{1} u_{1}^{x_{1}}=u_{2}^{x_{2}} v_{2}\right.$ in $\left.G\right\}$ is semilinear. Moreover, one can compute a semi-linear representation whose magnitude is bounded by $p\left(\left|u_{1}\right|+\left|v_{1}\right|+\left|u_{2}\right|+\left|v_{2}\right|\right)$ for a fixed polynomial $p(n)$.

Proof. Let $S:=\left\{\left(x_{1}, x_{2}\right) \in \mathbb{N} \times \mathbb{N} \mid v_{1} u_{1}^{x_{1}}=u_{2}^{x_{2}} v_{2}\right.$ in $\left.G\right\}$. We will define an NFA $\mathcal{A}$ over the alphabet $\left\{a_{1}, a_{2}\right\}$ such that the Parikh image of $L(A)$ is $S$. Moreover, the number of states of $\mathcal{A}$ is polynomial in $\left|u_{1}\right|+\left|u_{2}\right|+\left|v_{1}\right|+\left|v_{2}\right|$. This allows us to apply Theorem 8.2. We will allow transitions that are labelled with words (having length polynomial in $\left.\left|u_{1}\right|+\left|u_{2}\right|+\left|v_{1}\right|+\left|v_{2}\right|\right)$. Moreover, instead of writing in the transitions these words, we write their Parikh images (so, for instance, a transition $p \stackrel{a_{1}^{2} a_{2}^{3}}{\longrightarrow} q$ is written as $p \stackrel{(2,3)}{\longrightarrow} q$.

Let $\ell_{i}=\left|u_{i}\right|$ and $m_{i}=\left|v_{i}\right|$. Take the constant $\kappa$ from Lemma 3.3 and define $N_{1}=\lambda\left(m_{1}+2 \delta+\kappa\right)+\epsilon$ and $N_{2}=\lambda\left(m_{2}+2 \delta+\kappa\right)+\epsilon$. We split the set $S$ into two parts:

- $S_{1}=S \cap\left\{\left(n_{1}, n_{2}\right) \in \mathbb{N} \times \mathbb{N} \mid n_{1}<\left(N_{1}+N_{2}\right) / \ell_{1}\right\}$

- $S_{2}=S \cap\left\{\left(n_{1}, n_{2}\right) \in \mathbb{N} \times \mathbb{N} \mid n_{1} \geq\left(N_{1}+N_{2}\right) / \ell_{1}\right\}$

For all $\left(n_{1}, n_{2}\right) \in S_{1}$ we have $\left|u_{1}^{n_{1}}\right|=n_{1} \ell_{1}<N_{1}+N_{2}$. Hence, $\left|\operatorname{shlex}\left(u_{2}^{n_{2}}\right)\right|=$ $\left|\operatorname{shlex}\left(v_{1} u_{1}^{n_{1}} v_{2}^{-1}\right)\right|<N_{1}+N_{2}+m_{1}+m_{2}$. Since $u_{2}^{n_{2}}$ is $(\lambda, \epsilon)$-quasigeodesic we get $\left|u_{2}^{n_{2}}\right|=n_{2} \ell_{2}<\lambda\left(N_{1}+N_{2}+m_{1}+m_{2}\right)+\epsilon$, i.e., $n_{2}<\left(\lambda\left(N_{1}+N_{2}+m_{1}+m_{2}\right)+\epsilon\right) / \ell_{2}$. 


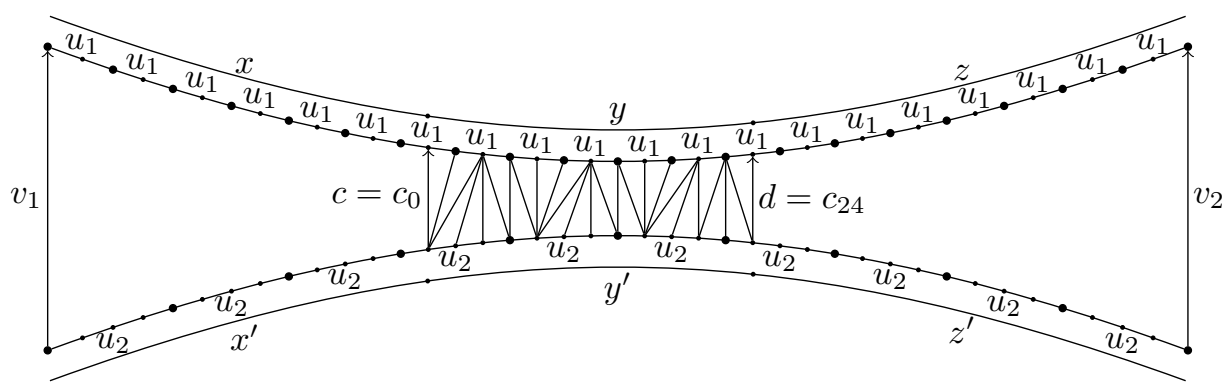

Figure 4. Example for the construction from the proof of Lemma 8.3

Hence, the set $S_{1}$ is finite and has a semilinear representation where all numbers are bounded by $\mathcal{O}\left(m_{1}+m_{2}\right)$.

We now deal with pairs $\left(n_{1}, n_{2}\right) \in S_{2}$, where $v_{1} u_{1}^{n_{1}}=u_{2}^{n_{2}} v_{2}$ in $G$ and $n_{1} \geq\left(N_{1}+\right.$ $\left.N_{2}\right) / \ell_{1}$, i.e., $\left|u_{1}^{n_{1}}\right| \geq N_{1}+N_{2}$. Consider such a pair $\left(n_{1}, n_{2}\right)$ and the quasigeodesic rectangle consisting of the four paths $Q_{1}=P\left[v_{1}\right], P_{1}=v_{1} \cdot P\left[u_{1}^{n_{1}}\right], P_{2}=P\left[u_{2}^{n_{2}}\right]$, and $Q_{2}=u_{2}^{n_{2}} \cdot P\left[v_{2}\right]$. We factorize the word $u_{1}^{n_{1}}$ as $u_{1}^{n_{1}}=x y z$ with $|x|=N_{1}$ and $|z|=N_{2}$. By Lemma 3.3 we can factorize $u_{2}^{n_{2}}$ as $u_{2}^{n_{2}}=x^{\prime} y^{\prime} z^{\prime}$ such that there exist $c, d \in \mathcal{B}_{2 \delta+2 \kappa}(1)$ with $v_{1} x=x^{\prime} c$ and $d z=z^{\prime} v_{2}$ in $G$, see Figure 4 (where $n_{1}=20$, $n_{2}=10, \ell_{1}=2$ and $\left.\ell_{2}=4\right)$. Since $u_{2}^{n_{2}}$ is $(\lambda, \epsilon)$-quasigeodesic, we have

$$
\begin{aligned}
& \left|x^{\prime}\right| \leq \lambda\left(m_{1}+|x|+2 \delta+2 \kappa\right)+\epsilon=\lambda\left(m_{1}+N_{1}+2 \delta+2 \kappa\right)+\epsilon, \\
& \left|z^{\prime}\right| \leq \lambda\left(m_{2}+|z|+2 \delta+2 \kappa\right)+\epsilon=\lambda\left(m_{2}+N_{2}+2 \delta+2 \kappa\right)+\epsilon .
\end{aligned}
$$

Consider now the subpath $P_{1}^{\prime}$ of $P_{1}$ from $P_{1}(|x|)$ to $P_{1}\left(n_{1} \ell_{1}-|z|\right)$ and the subpath $P_{2}^{\prime}$ of $P_{2}$ from $P_{2}\left(\left|x^{\prime}\right|\right)$ to $P_{2}\left(n_{2} \ell_{2}-\left|z^{\prime}\right|\right)$. These are the paths labelled with $y$ and $y^{\prime}$, respectively, in Figure 4. By Lemma 3.2 these paths asynchronously $\gamma$-fellow travel, where $\gamma:=K(\delta, \lambda, \epsilon, 2 \delta+2 \kappa)$ is a constant. In Figure 4 this is visualized by the part between the $c$-labelled edge and the $d$-labelled edge. W.l.o.g. we assume that $\gamma \geq 2 \delta+2 \kappa$.

We now define the NFA $\mathcal{A}$ over the alphabet $\left\{a_{1}, a_{2}\right\}$ (recall the we replace edge labels from $\left\{a_{1}, a_{2}\right\}^{*}$ by their Parikh images). The state set of $\mathcal{A}$ is

$$
Q=\left\{q_{0}, q_{f}\right\} \cup\left\{(i, b, j) \mid 0 \leq i<\ell_{1}, 0 \leq j<\ell_{2}, b \in \mathcal{B}_{\gamma}(1)\right\} .
$$

The unique initial state is $q_{0}$ and the unique final state is $q_{f}$. To define the transitions of $\mathcal{A}$ set $p=\left\lfloor N_{1} / \ell_{1}\right\rfloor=\left\lfloor|x| /\left|u_{1}\right|\right\rfloor, r=N_{1} \bmod \ell_{1}=|x| \bmod \left|u_{1}\right|$, $s=\left\lceil N_{2} / \ell_{1}\right\rceil=\left\lceil|z| /\left|u_{1}\right|\right\rceil, t=-N_{2} \bmod \ell_{1}=-|z| \bmod \left|u_{1}\right|$. Thus, we have $x=u_{1}^{p} u_{1}[: r]$ and $z=u_{1}^{s}[t+1:]$. There are the following types of transitions (transitions without a label are implicitly labelled by the zero vector $(0,0)$ ), where $0 \leq i<\ell_{1}, 0 \leq j<\ell_{2}, b, b^{\prime} \in \mathcal{B}_{\gamma}(1)$.

(1) $q_{0} \stackrel{\left(p, p^{\prime}\right)}{\longrightarrow}\left(r, c, r^{\prime}\right)$ if there exists a number $0 \leq k \leq \lambda\left(m_{1}+N_{1}+2 \delta+2 \kappa\right)+\epsilon$ (this is the possible range for the length of $x^{\prime}$ in (1) ) such that $p^{\prime}=\left\lfloor k / \ell_{2}\right\rfloor$, $r^{\prime}=k \bmod \ell_{2}$, and $v_{1} u_{1}^{p} u_{1}[: r]=u_{2}^{p^{\prime}} u_{2}\left[: r^{\prime}\right] c$ in $G$.

(2) $(i, b, j) \rightarrow\left(i+1, b^{\prime}, j\right)$ if $i+1<\ell_{1}$ and $b u_{1}[i+1]=b^{\prime}$ in $G$.

(3) $\left(\ell_{1}-1, b, j\right) \stackrel{(1,0)}{\longrightarrow}\left(0, b^{\prime}, j\right)$ if $b u_{1}\left[\ell_{1}\right]=b^{\prime}$ in $G$.

(4) $(i, b, j) \rightarrow\left(i, b^{\prime}, j+1\right)$ if $j+1<\ell_{2}$ and $b=u_{2}[j+1] b^{\prime}$ in $G$.

(5) $\left(i, b, \ell_{2}-1\right) \stackrel{(0,1)}{\longrightarrow}\left(i, b^{\prime}, 0\right)$ if $b=u_{2}\left[\ell_{2}\right] b^{\prime}$ in $G$.

(6) $\left(t, d, t^{\prime}\right) \stackrel{\left(s, s^{\prime}\right)}{\longrightarrow} q_{f}$ if there exists a number $0 \leq k \leq \lambda\left(m_{2}+N_{2}+2 \delta+2 \kappa\right)+\epsilon$ (this is the possible range for the length of $z^{\prime}$ in (2) ) such that $s^{\prime}=\left\lceil k / \ell_{2}\right\rceil$, $t^{\prime}=-k \bmod \ell_{2}$, and $d u_{1}[t+1:] u_{1}^{s}=u_{2}\left[t^{\prime}+1:\right] u_{2}^{s^{\prime}} v_{2}$ in $G$. 
The construction is best explained using the example in Figure 4. As mentioned above, the vertical lines between $c=c_{0}$ and $d=c_{24}$ represent the asynchronous $\gamma$ fellow travelling. The vertical lines are labelled with group elements $c_{0}, c_{1}, \ldots, c_{23}, c_{24} \in$ $\mathcal{B}_{\gamma}(1)$ from left to right. In order to not overload the figure we only show $c_{0}$ and $c_{24}$. Note that $x=u_{1}^{6} u_{1}[1], x^{\prime}=u_{2}^{3} u_{2}[1], z=u_{1}^{8}[2:], z^{\prime}=u_{2}^{4}[2:]$. Basically, the NFA $\mathcal{A}$ moves the vertical edges from left to right and thereby stores (i) the label $c_{i}$ of the vertical edge, (ii) the position in the current $u_{2}$-factor where the vertical edge starts (position 0 means that we have just completed a $u_{2}$-factor), and (iii) the position in the current $u_{1}$-factor where the vertical edge ends. If a $u_{1}$-factor (resp., $u_{2}$-factor) is completed then the automaton makes a $(1,0)$-labelled (resp., $(0,1)$-labelled) transition. The automaton run corresponding to Figure 4 is:

$$
\begin{aligned}
q_{0} \stackrel{(6,3)}{\longrightarrow} & \left(1, c_{0}, 1\right) \stackrel{(1,0)}{\longrightarrow}\left(0, c_{1}, 1\right) \rightarrow\left(1, c_{2}, 1\right) \rightarrow\left(1, c_{3}, 2\right) \rightarrow\left(1, c_{4}, 3\right) \stackrel{(0,1)}{\longrightarrow} \\
& \left(1, c_{5}, 0\right) \stackrel{(1,0)}{\longrightarrow}\left(0, c_{6}, 0\right) \rightarrow\left(0, c_{7}, 1\right) \rightarrow\left(1, c_{8}, 1\right) \stackrel{(1,0)}{\longrightarrow}\left(0, c_{9}, 1\right) \rightarrow \\
& \left(1, c_{10}, 1\right) \rightarrow\left(1, c_{11}, 2\right) \rightarrow\left(1, c_{12}, 3\right) \stackrel{(0,1)}{\longrightarrow}\left(1, c_{13}, 0\right) \stackrel{(1,0)}{\longrightarrow}\left(0, c_{14}, 0\right) \rightarrow \\
& \left(0, c_{15}, 1\right) \rightarrow\left(1, c_{16}, 1\right) \stackrel{(1,0)}{\longrightarrow}\left(0, c_{17}, 1\right) \rightarrow\left(1, c_{18}, 1\right) \rightarrow\left(1, c_{19}, 2\right) \rightarrow \\
& \left(1, c_{20}, 3\right) \stackrel{(1,0)}{\longrightarrow}\left(0, c_{21}, 3\right) \stackrel{(0,1)}{\longrightarrow}\left(0, c_{22}, 0\right) \rightarrow\left(0, c_{23}, 1\right) \rightarrow\left(1, c_{24}, 1\right) \stackrel{(8,4)}{\longrightarrow} q_{f}
\end{aligned}
$$

With the above intuition it is straightforward to show that the Parikh image of $L(\mathcal{A})$ is indeed $S_{2}$. Also note that the number of states of $\mathcal{A}$ is bounded by $\mathcal{O}\left(\ell_{1} \ell_{2}\right)$. The statement of the lemma then follows directly from Theorem 8.2 .

8.2. Reduction to quasi-geodesic knapsack expressions. Let us call a knapsack expression $E=u_{1}^{x_{1}} v_{1} u_{2}^{x_{2}} v_{2} \cdots u_{k}^{x_{k}} v_{k}$ over $G(\lambda, \epsilon)$-quasigeodesic if all words $u_{1}, \ldots, u_{k}, v_{1}, \ldots, v_{k}$ are geodesic and for all $1 \leq i \leq k$ and all $n \geq 0$ the word $u_{i}^{n}$ is $(\lambda, \epsilon)$-quasigeodesic. We say that $E$ has infinite order, if all $u_{i}$ represent group elements of infinite order. The goal of this section is to reduce a knapsack expression to a finite number (in fact, exponentially many) of $(\lambda, \epsilon)$-quasigeodesic knapsack expressions of infinite order for certain constants $\lambda, \epsilon$ :

Proposition 8.4. There exist fixed constants $\lambda, \epsilon$ such that from a given knapsack expression $E$ over $G$ one can compute a finite list of knapsack expressions $E_{i}(i \in I)$ over $G$ such that

$$
\operatorname{sol}(E)=\bigcup_{i \in I}\left(\left(m_{i} \cdot \operatorname{sol}\left(E_{i}\right)+d_{i}\right) \oplus \mathcal{F}_{i}\right)
$$

where the following additional properties hold:

- every $\mathcal{F}_{i}$ is a semilinear subset of $\mathbb{N}^{Y}$ for a subset $Y \subseteq X_{E}$,

- the magnitude of every $\mathcal{F}_{i}$ is bounded by a constant that only depends on $G$,

- every $E_{i}$ is a $(\lambda, \epsilon)$-quasigeodesic knapsack expression of infinite order with variables from $Z:=X_{E} \backslash Y$,

- the size of every $E_{i}$ is bounded by $\mathcal{O}(|E|)$, and

- all $m_{i}$ and $d_{i}$ are vectors from $\mathbb{N}^{Z}$ where all entries are bounded by a constant that only depends on $G$ (here, $m_{i} \cdot \operatorname{sol}\left(E_{i}\right)=\left\{m_{i} \cdot z \mid z \in \operatorname{sol}(E)\right\}$ and $m_{i} \cdot z$ is the pointwise multiplication of the vectors $m_{i}$ and $\left.z\right)$.

Once Proposition 8.4 is shown, we can conclude the proof of Theorem 8.1 by showing that all sets $\operatorname{sol}\left(E_{i}\right)$ are semilinear and that their magnitudes are bounded by $p\left(\left|E_{i}\right|\right)$ for a fixed polynomial $p(n)$. This will be achieved in the next section.

For the proof of Proposition 8.4 we mainly build on results from 3 . We fix the constants $L=34 \delta+2$ and $K=\left|\mathcal{B}_{4 \delta}(1)\right|^{2}$. 
Lemma 8.5 (c.f. [3, Lemma 3.1]). Let $u=u_{1} u_{2}$ be shortlex reduced, where $\left|u_{1}\right| \leq$ $\left|u_{2}\right| \leq\left|u_{1}\right|+1$. Let $\tilde{u}=\operatorname{shlex}\left(u_{2} u_{1}\right)$. If $|\tilde{u}| \geq 2 L+1$ then for every $n \geq 0$, the word $\tilde{u}^{n}$ is L-local $(1,2 \delta)$-quasigeodesic.

The following lemma is not stated explicitly in [3] but is shown in Section 3.2 (where the main argument is attributed to Delzant).

Lemma 8.6 (c.f. $[\underline{3}$ ). Let $u$ be geodesic such that $|u| \geq 2 L+1$ and for every $n \geq 0$, the word $u^{n}$ is L-local $(1,2 \delta)$-quasigeodesic. Then one can compute $c \in \mathcal{B}_{4 \delta}(1)$ and an integer $1 \leq m \leq K$ such that $\left(\operatorname{shlex}\left(c^{-1} u^{m} c\right)\right)^{n}$ is geodesic for all $n \geq 0$.

Proof of Proposition 8.4. We set $\lambda=N(2 L+1)$ and $\epsilon=2 N^{2}(2 L+1)^{2}+2 N(2 L+1)$, where $N=\left|\mathcal{B}_{2 \delta}(1)\right|$. Consider a knapsack expression $E=u_{1}^{x_{1}} v_{1} u_{2}^{x_{2}} v_{2} \cdots u_{k}^{x_{k}} v_{k}$. We can assume that every $u_{i}$ is shortlex reduced. Let $g_{i} \in G$ be the group element represented by the word $u_{i}$.

Step 1. In this first step we show how to reduce to the case where all $g_{i}$ have infinite order. In a hyperbolic group $G$ the order of torsion elements is bounded by a fixed constant that only depends on $G$, see also the proof of [22, Theorem 6.7]). This allows to check for each $g_{i}$ whether it has finite order, and to compute the order in the positive case. Let $Y \subseteq\left\{x_{1}, \ldots, x_{k}\right\}$ be those variables $x_{i}$ such that $g_{i}$ has finite order. For $x_{i} \in Y$ let $o_{i}<\infty$ be the order of $g_{i}$. Let $\mathcal{F}$ be the set of mappings $f: Y \rightarrow \mathbb{N}$ such that $0 \leq f\left(x_{i}\right)<o_{i}$ for all $x_{i} \in Y$. For every such mapping $f \in \mathcal{F}$ let $E_{f}$ be the knapsack expression that is obtained from $E$ by replacing for every $x_{i} \in Y$ the power $u_{i}^{x_{i}}$ by $u_{i}^{f\left(x_{i}\right)}$ (which is merged with the word $v_{i}$ ). Moreover, let $\mathcal{F}_{f}$ be the set of all mappings $g: Y \rightarrow \mathbb{N}$ such that $g\left(x_{i}\right) \equiv f\left(x_{i}\right)$ mod $o_{i}$ for every $x_{i} \in Y$. Then the set sol $(E)$ can be written as

$$
\operatorname{sol}(E)=\bigcup_{f \in \mathcal{F}} \operatorname{sol}\left(E_{f}\right) \oplus \mathcal{F}_{f} .
$$

Note that $\mathcal{F}_{f}$ is a semilinar set of magnitude $\mathcal{O}(1)$.

Step 2. We now consider a knapsack expression from $\mathcal{F}_{f}$. To simplify notation, we denote this expression again with $E=u_{1}^{x_{1}} v_{1} u_{2}^{x_{2}} v_{2} \cdots u_{k}^{x_{k}} v_{k}$. For every $i$, the group element $g_{i}$ represented by $u_{i}$ has infinite order. We factorize $u_{i}$ uniquely as $u_{i}=u_{i, 1} u_{i, 2}$ where $\left|u_{i, 1}\right| \leq\left|u_{i, 2}\right| \leq\left|u_{i, 1}\right|+1$, and let $\tilde{u}_{i}=\operatorname{shlex}\left(u_{i, 2} u_{i, 1}\right)$. Note that $\left|\tilde{u}_{i}\right| \leq\left|u_{i}\right|$. Let $\tilde{g}_{i}$ be the group element represented by $\tilde{u}_{i}$. Since $\tilde{g}_{i}$ is conjugated to $g_{i}$, also $\tilde{g}_{i}$ has infinite order. By Lemma 3.1, for every $n \geq 0$, the word $\tilde{u}_{i}^{n}$ is $\left(\lambda_{i}, \epsilon_{i}\right)$-quasigeodesic for $\lambda_{i}=N\left|\tilde{u}_{i}\right|, \epsilon_{i}=2 N^{2}\left|\tilde{u}_{i}\right|^{2}+2 N\left|\tilde{u}_{i}\right|$. If $\left|\tilde{u}_{i}\right|<2 L+1$ then $\tilde{u}_{i}^{n}$ is $(\lambda, \epsilon)$-quasigeodesic for the constants $\lambda$ and $\epsilon$ defined at the beginning of the proof. We then replace $u_{i}^{x_{i}}$ by $u_{i, 1} \tilde{u}_{i}^{x_{i}} u_{i, 1}^{-1}$. Note that for every $n \geq 0$, $u_{i, 1} \tilde{u}_{i}^{n} u_{i, 1}^{-1}=u_{i, 1}\left(u_{i, 2} u_{i, 1}\right)^{n} u_{i, 1}^{-1}=\left(u_{i, 1} u_{i, 2}\right)^{n}=u_{i}^{n}$ in $G$.

Now assume that $\left|\tilde{u}_{i}\right| \geq 2 L+1$. By Lemma 8.5, $\tilde{u}_{i}^{n}$ is $L$-local $(1,2 \delta)$-quasigeodesic for every $n \geq 0$. By Lemma [8.6, one can compute $c_{i} \in \mathcal{B}_{4 \delta}(1)$ and an integer $1 \leq m_{i} \leq K$ such that $\left(\operatorname{shlex}\left(c_{i}^{-1} \tilde{u}_{i}^{m_{i}} c_{i}\right)\right)^{n}$ is geodesic (and hence $(1,0)$ quasigeodesic) for all $n \geq 0$. We then produce for every number $0 \leq d_{i} \leq m_{i}-1$ a new knapsack instance by replacing $u_{i}^{x_{i}}$ by $u_{i, 1} \tilde{u}_{i}^{d_{i}} c_{i}\left(\operatorname{shlex}\left(c_{i}^{-1} \tilde{u}_{i}^{m_{i}} c_{i}\right)\right)^{x_{i}} c_{i}^{-1} u_{i, 1}^{-1}$. To make the description of the resulting knapsack expression more uniform we set $m_{i}=1$ and $c_{i}=1$ in case $\left|\tilde{u}_{i}\right|<2 L+1$. Then, the replacement of $u_{i}^{x_{i}}$ by $u_{i, 1} \tilde{u}_{i}^{x_{i}} u_{i, 1}^{-1}$ in case $\left|\tilde{u}_{i}\right|<2 L+1$ is the same as the one for the case $\left|\tilde{u}_{i}\right| \geq 2 L+1$. Let $m:\left\{x_{1}, \ldots, x_{k}\right\} \rightarrow \mathbb{N}$ be the mapping with $m\left(x_{i}\right)=m_{i}$.

From the above discussion, we obtain a finite set of $(\lambda, \epsilon)$-quasigeodesic knapsack expressions $E_{d}$ that are parameterized by a mapping $d:\left\{x_{1}, \ldots, x_{k}\right\} \rightarrow \mathbb{N}$ with 


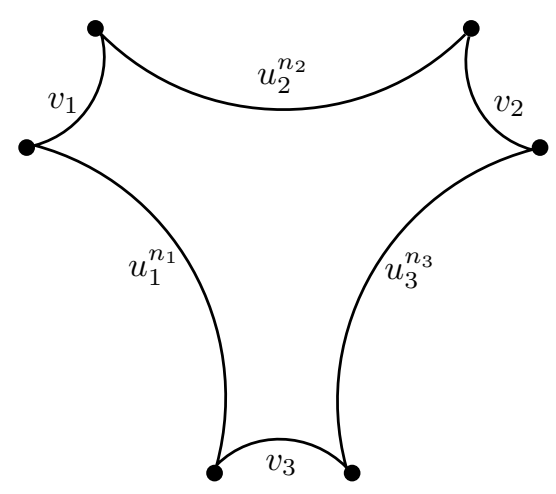

FiguRE 5 . The $2 k$-gon for $k=3$ from the proof of Theorem 8.1

$0 \leq d\left(x_{i}\right)<m_{i}$ for all $1 \leq i \leq k$. Let $\mathcal{D}$ be the set of all such mappings. We then have

$$
\operatorname{sol}(E)=\bigcup_{d \in \mathcal{D}}\left(m \cdot \operatorname{sol}\left(E_{d}\right)+d\right) .
$$

Note that the magnitude of every $E_{d}$ is bounded linearly in the magnitude of $E$.

Finally, the statement of the proposition is directly obtained by combining the above steps 1 and 2 .

8.3. Proof of Theorem 8.1. We now come to the proof of Theorem 8.1. Consider a knapsack expression $E=u_{1}^{x_{1}} v_{1} u_{2}^{x_{2}} v_{2} \cdots u_{k}^{x_{k}} v_{k}$. We can assume that all $u_{i}, v_{i}$ are geodesic. By Proposition 8.4 we can moreover assume that for all $1 \leq i \leq k, u_{i}$ represents a group element of infinite order and that $u_{i}^{n}$ is $(\lambda, \epsilon)$-quasigeodesic for all $n \geq 0$, where $\lambda, \epsilon$ are fixed constants. We want to show that $\operatorname{sol}(E)$ is semilinear and has a magnitude that is polynomially bounded by $|E|$.

For the case $k=1$ we have to consider all natural numbers $n$ with $u_{1}^{n}=v_{1}^{-1}$ in $G$. Since $u_{1}$ represents a group element of infinite order there is at most one such $n$. Moreover, since $u_{i}^{n}$ is $(\lambda, \epsilon)$-quasigeodesic, such an $n$ has to satisfy $\left|u_{1}\right| \cdot n \leq \lambda\left|v_{1}\right|+\epsilon$, which yields a linear bound on $n$.

For the case $k=2$ we can directly use Proposition 8.3 . Now assume that $k \geq 3$. We want to show that the set $\operatorname{sol}(E)$ is a semilinear subset of $\mathbb{N}^{k}$ (later we will consider the magnitude of $\operatorname{sol}(E))$. For this we construct a Presburger formula with free variables $x_{1}, \ldots, x_{k}$ that is equivalent to $E=1$. We do this by induction on the depth $k$. Therefore, we can use in our Presburger formula also knapsack equations of the form $F=1$, where $F$ has depth at most $k-1$.

It suffices to construct a Presburger formula for $\operatorname{sol}(E) \cap(\mathbb{N} \backslash\{0\})^{k}$. Note that $E=1$ is equivalent to $\bigvee_{I \subseteq\{1, \ldots, k\}}\left(E_{I}=1 \wedge \bigwedge_{i \in I} x_{i}>0\right)$, where $E_{I}$ is obtained from $E$ by removing for every $i \notin I$ the power $u_{i}^{x_{i}}$.

Consider a tuple $\left(n_{1}, \ldots, n_{k}\right) \in \operatorname{sol}(E) \cap(\mathbb{N} \backslash\{0\})^{k}$ and the corresponding $2 k$-gon that is defined by the $(\lambda, \epsilon)$-quasigeodesic paths $P_{i}=\left(u_{1}^{n_{1}} v_{1} \cdots u_{i-1}^{n_{i-1}} v_{i-1}\right) \cdot P\left[u_{i}^{n_{i}}\right]$ and the geodesic paths $Q_{i}=\left(u_{1}^{n_{1}} v_{1} \cdots u_{i}^{n_{i}}\right) \cdot P\left[v_{i}\right]$, see Figure 5 for the case $k=3$. Since all paths $P_{i}$ and $Q_{i}$ are $(\lambda, \epsilon)$-quasigeodesic, we can apply [22, Lemma 6.4]: Every side of the $2 k$-gon is contained in the $h$-neighborhoods of the other sides, where $h=\xi+\xi \log (2 k)$ for a constant $\xi$ that only depends on the constants $\delta, \lambda, \varepsilon$.

Let us now consider the side $P_{2}$ of the quasigeodesic $(2 k)$-gon. It is labelled with $u_{2}^{x_{2}}$. Its neighboring sides are $Q_{1}$ and $Q_{2}$, which are labelled with $v_{1}$ and $v_{3}$, respectively. We distinguish several cases. In each case we cut the $2 k$-gon into smaller pieces along paths of length $\leq 2 h+1$ (length $h$ in some cases), and these smaller pieces will correspond to knapsack expressions of depth $<k$. This is done 


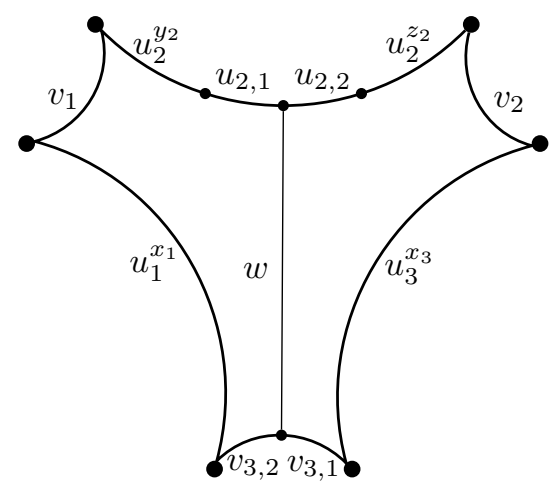

Figure 6. Case 1.1 from the proof of Theorem 8.1

until all knapsack expressions have depth at most two. When we speak of a point on the $2 k$-gon, we mean a node of the Cayley graph (i.e., an element of the group $G)$ and not a point in the interior of an edge. Moreover, when we speak of the successor point of a point $p$, we refer to the clockwise order on the $2 k$-gon, where the sides are traversed in the order $P_{1}, Q_{1}, \ldots, P_{k}, Q_{k}$. We now distinguish the following cases:

Case 1: There is a point $p \in P_{2}$ that has distance at most $h$ from a point $q$ that does not belong to $P_{1} \cup Q_{1} \cup Q_{2} \cup P_{3}$. Thus $q$ must belong to one of the paths $Q_{3}, P_{4}, \ldots Q_{k-1}, P_{k}, Q_{k}$. Let $w$ be a geodesic word of length at most $h$ that labels a path from $p$ to $q$. There are two subcases:

Case 1.1: $q$ belongs to the paths $Q_{i}$, where $3 \leq i \leq k$. The situation is shown in Figure 6. We construct two new knapsack expressions $F_{t}$ and $G_{t}$ for all tuples $t=\left(w, u_{2,1}, u_{2,2}, v_{i, 1}, v_{i, 2}\right)$ such that $w \in \Sigma^{*}$ is of length at most $h, u_{2}=u_{2,1} u_{2,2}$ and $v_{i}=v_{i, 1} v_{i, 2}$ :

$$
\begin{aligned}
F_{t} & =u_{1}^{x_{1}} v_{1} u_{2}^{y_{2}}\left(u_{2,1} w v_{i, 2}\right) u_{i+1}^{x_{i+1}} v_{i+1} \cdots u_{k}^{x_{k}} v_{k} \text { and } \\
G_{t} & =u_{2,2} u_{2}^{z_{2}} v_{2} u_{3}^{x_{3}} v_{3} \cdots u_{i}^{x_{i}}\left(v_{i, 1} w^{-1}\right)
\end{aligned}
$$

Here $y_{2}$ and $z_{2}$ are new variables. Note that $F_{t}$ and $G_{t}$ have depth at most $k-1$. Moreover, let $A_{1.1}$ be the following formula, where $t$ ranges over all tuples of the above form:

$$
A_{1.1}=\bigvee_{t} \exists y_{2}, z_{2}: x_{2}=y_{2}+1+z_{2} \wedge F_{t}=1 \wedge G_{t}=1
$$

Case 1.2: $q$ belongs to the path $P_{i}$, where $4 \leq i \leq k$ (this case can only occur if $k \geq 4)$. This case is analogous to Case 1.1. We only have to split $u_{i}^{x_{i}}$ as $u_{i}^{y_{i}}\left(u_{i, 1} u_{i, 2}\right) u_{i}^{z_{i}}$ (as we do for $u_{2}^{x_{2}}$ ). We construct two new knapsack expressions $F_{t}$ and $G_{t}$ for all tuples $t=\left(w, u_{2,1}, u_{2,2}, u_{i, 1}, u_{i, 2}\right)$ such that $w \in \Sigma^{*}$ is of length at most $h, u_{2}=u_{2,1} u_{2,2}$ and $u_{i}=u_{i, 1} u_{i, 2}$ :

$$
\begin{aligned}
F_{t} & =u_{1}^{x_{1}} v_{1} u_{2}^{y_{2}}\left(u_{2,1} w u_{i, 2}\right) u_{i}^{z_{i}} v_{i} u_{i+1}^{x_{i+1}} v_{i+1} \cdots u_{k}^{x_{k}} v_{k} \text { and } \\
G_{t} & =u_{2,2} u_{2}^{z_{2}} v_{2} u_{3}^{x_{3}} v_{3} \cdots u_{i-1}^{x_{i-1}} v_{i-1} u_{i}^{y_{i}}\left(u_{i, 1} w^{-1}\right)
\end{aligned}
$$

Here $y_{2}, z_{2}, y_{i}, z_{i}$ are new variables. Note that $F_{t}$ and $G_{t}$ have depth at most $k-1$. Moreover, let $A_{1.2}$ be the following formula, where $t$ ranges over all tuples of the above form:

$$
A_{1.2}=\bigvee_{t} \exists y_{2}, z_{2}, y_{i}, z_{i}: x_{2}=y_{2}+1+z_{2} \wedge x_{i}=y_{i}+1+z_{i} \wedge F_{t}=1 \wedge G_{t}=1
$$




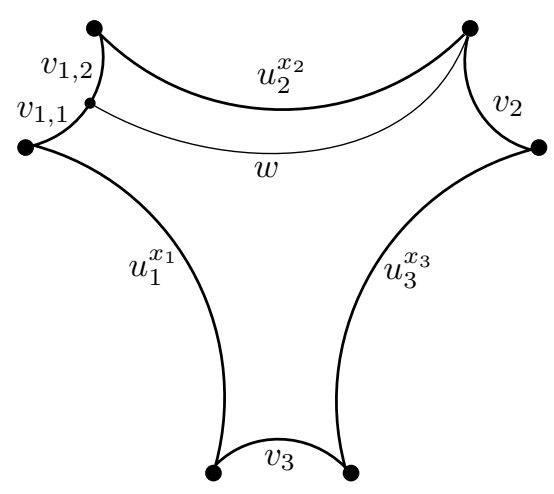

Figure 7. Case 2.1 from the proof of Theorem 8.1

Case 2: Every point on $P_{2}$ that has distance at most $h$ from a point on $P_{1} \cup Q_{1} \cup$ $Q_{2} \cup P_{3}$.

Case 2.1: The end point of $P_{2}$ (i.e., the point connecting $P_{2}$ with $Q_{2}$ ) has distance at most $h$ from a point on $Q_{1}$, see Figure 7 For all tuples $t=\left(w, v_{1,1}, v_{1,2}\right)$ such that $w \in \Sigma^{*}$ is of length at most $h$ and $v_{1}=v_{1,1} v_{1,2}$ we construct two new knapsack expressions

$$
F_{t}=u_{2}^{x_{2}}\left(w v_{1,2}\right) \text { and } G_{t}=u_{1}^{x_{1}}\left(v_{1,1} w^{-1} v_{2}\right) u_{3}^{x_{3}} v_{3} \cdots u_{k}^{x_{k}} v_{k}
$$

and the formula

$$
A_{2.1}=\bigvee_{t} F_{t}=1 \wedge G_{t}=1,
$$

where $t$ ranges over all tuples of the above form. Note that $F_{t}$ has depth one and $G_{t}$ has depth $k-1$.

Case 2.2: The end point of $P_{2}$ (i.e., the point connecting $P_{2}$ with $Q_{2}$ ) has distance at most $h$ from a point on $P_{1}$, see Figure 8 . For all tuples $t=\left(w, u_{1,1}, u_{1,2}\right)$ such that $w \in \Sigma^{*}$ is of length at most $h$ and $u_{1}=u_{1,1} u_{1,2}$, we construct two new knapsack expressions

$$
F_{t}=u_{1}^{z_{1}} v_{1} u_{2}^{x_{2}}\left(w u_{1,2}\right) \text { and } G_{t}=u_{1}^{y_{1}}\left(u_{1,1} w^{-1} v_{2}\right) u_{3}^{x_{3}} v_{3} \cdots u_{k}^{x_{k}} v_{k}
$$

and the formula

$$
A_{2.2}=\bigvee_{t} \exists y_{1}, z_{1}: x_{1}=y_{1}+1+z_{1} \wedge F_{t}=1 \wedge G_{t}=1
$$

where $t$ ranges over all tuples of the above form. Note that $F_{t}$ has depth two and $G_{t}$ has depth $k-1$.

If on the other hand the end point of $P_{2}$ has distance $>h$ from all points on $P_{1} \cup Q_{1}$, then there must be two points $p_{1}, p_{2}$ on $P_{2}$ such that $p_{2}$ is the successor point of $p_{1}$ when travelling along $P_{2}$ (i.e., $d\left(p_{1}, p_{2}\right)=1$ ), and $p_{1}$ has distance at most $h$ from a point $q_{1} \in P_{1} \cup Q_{1}$, while $p_{2}$ has distance at most $h$ from a point on $q_{2} \in Q_{2} \cup P_{3}$. Thus, the distance between $q_{1}$ and $q_{2}$ is at most $2 h+1$. Let $w$ be a word that labels a geodesic path from $q_{1}$ to $q_{2}$ (thus, $|w| \leq 2 h+1$ ). This leads to the following four subcases.

Case 2.3: $q_{1} \in Q_{1}$ and $q_{2} \in Q_{2}$, see Figure 9. This case is very similar to Case 2.1. For every tuple $t=\left(w, v_{1,1}, v_{1,2}, v_{2,1}, v_{2,2}\right)$ with $|w| \leq 2 h+1, v_{1}=v_{1,1} v_{1,2}$ and $v_{2}=v_{2,1} v_{2,2}$ we obtain two new knapsack expressions

$$
F_{t}=F_{t}=v_{1,2} u_{2}^{x_{2}}\left(v_{2,1} w\right) \text { and } G_{t}=u_{1}^{x_{1}}\left(v_{1,1} w^{-1} v_{2,2}\right) u_{3}^{x_{3}} v_{3} \cdots u_{k}^{x_{k}} v_{k}
$$




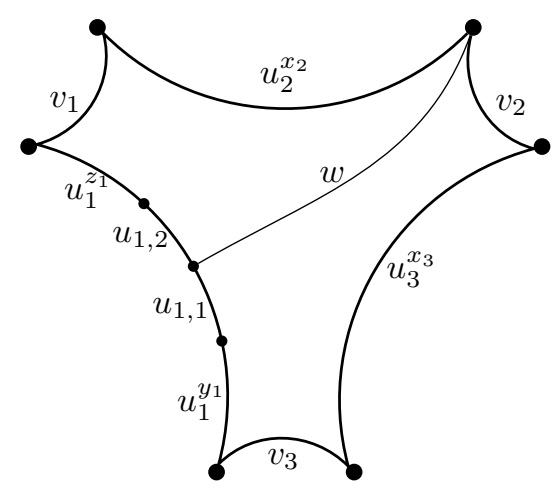

FiguRE 8. Case 2.2 from the proof of Theorem 8.1

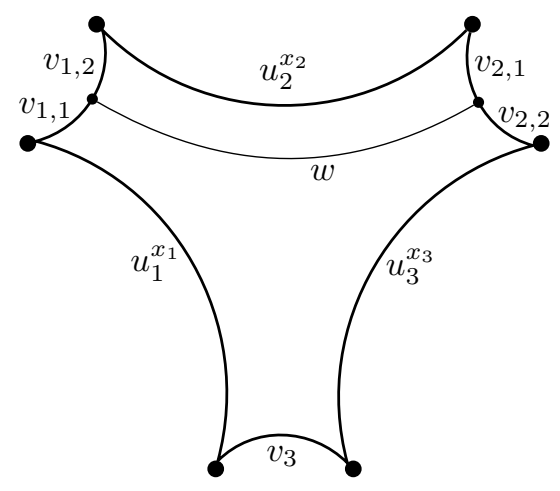

Figure 9. Case 2.3 from the proof of Theorem 8.1

and the formula

$$
A_{2.3}=\bigvee_{t} F_{t}=1 \wedge G_{t}=1,
$$

where $t$ ranges over all tuples of the above form.

Case 2.4: $q_{1} \in P_{1}$ and $q_{2} \in Q_{2}$, see Figure 10, This case is very similar to Case 2.2. For every tuple $t=\left(w, u_{1,1}, u_{1,2}, v_{2,1}, v_{2,2}\right)$ such that $|w| \leq 2 h+1, u_{1}=u_{1,1} u_{1,2}$, and $v_{2}=v_{2,1} v_{2,2}$ we obtain two new knapsack expressions

$$
F_{t}=u_{1,2} u_{1}^{z_{1}} v_{1} u_{2}^{x_{2}}\left(v_{2,1} w\right) \text { and } G_{t}=u_{1}^{y_{1}}\left(u_{1,1} w^{-1} v_{2,2}\right) u_{3}^{x_{3}} v_{3} \cdots u_{k}^{x_{k}} v_{k}
$$

and the formula

$$
A_{2.4}=\bigvee_{t} \exists y_{1}, z_{1}: x_{1}=y_{1}+1+z_{1} \wedge F_{t}=1 \wedge G_{t}=1,
$$

where $t$ ranges over all tuples of the above form.

Case 2.5: $q_{1} \in Q_{1}$ and $q_{2} \in P_{3}$. This case is analogous to Case 2.4.

Case 2.6: $q_{1} \in P_{1}$ and $q_{2} \in P_{3}$, see Figure [1], For every tuple

$$
\left(w_{1}, w_{2}, w, u_{1,1}, u_{1,2}, u_{2,1}, u_{2,2}, u_{3,1}, u_{3,2}\right)
$$

such that $|w| \leq 2 k+1,\left|w_{1}\right| \leq h,\left|w_{2}\right| \leq h+1, w=w_{1}^{-1} w_{2}$ in $G, u_{1}=u_{1,1} u_{1,2}$, $u_{2}=u_{2,1} u_{2,2}$, and $u_{3}=u_{3,1} u_{3,2}$ we obtain three new knapsack expressions

$$
\begin{aligned}
F_{t} & =u_{1}^{z_{1}} v_{1} u_{2}^{y_{2}}\left(u_{2,1} w_{1} u_{1,2}\right), \\
G_{t} & =u_{2}^{z_{2}} v_{2} u_{3}^{y_{3}}\left(u_{3,1} w_{2}^{-1} u_{2,2}\right) \text { and } \\
H_{t} & =u_{3}^{z_{3}} v_{3} u_{4}^{x_{4}} v_{4} \cdots u_{k}^{x_{k}} v_{k} u_{1}^{y_{1}}\left(u_{1,1} w u_{3,2}\right) .
\end{aligned}
$$




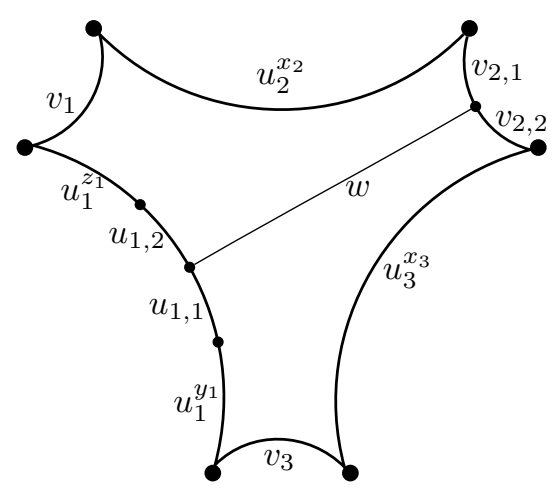

Figure 10. Case 2.4 from the proof of Theorem 8.1

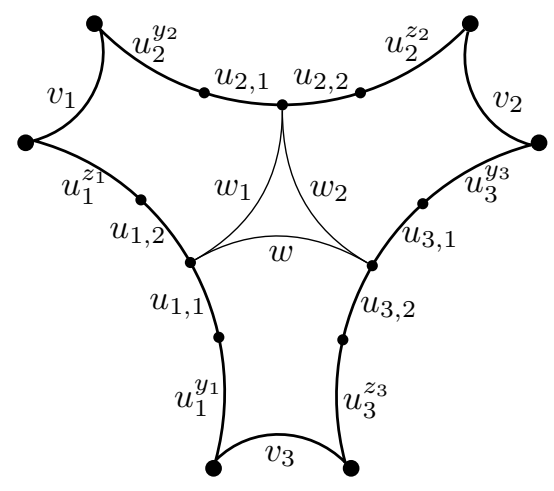

Figure 11. Case 2.6 from the proof of Theorem 8.1

and the formula

$$
A_{2.6}=\bigwedge_{t} \exists y_{1}, z_{1}, y_{2}, z_{2}, y_{3}, z_{3}: \bigwedge_{i=1}^{3} x_{i}=y_{i}+1+z_{i} \wedge F_{t}=1 \wedge G_{t}=1 \wedge H_{t}=1
$$

where $t$ ranges over all tuples of the above form. Note that $F_{t}$ and $G_{t}$ have depth 2 and that $H_{t}$ has depth $k-1$.

This concludes the construction of a Presburger formula for the set $\operatorname{sol}(E)$ and shows the semilinearity of $\operatorname{sol}(E)$. It remains to argue that the magnitude of sol $(E)$ is bounded polynomially in $|E|$. Iterating the above splitting procedure results in an exponentially large disjunction of conjunctive formulas of the form

$$
\exists y_{1}, \ldots, y_{m} \bigwedge_{i \in I} E_{i}=1 \bigwedge_{j \in J} z_{j}=z_{j}^{\prime}+z_{j}^{\prime \prime}+1
$$

where every $E_{i}$ is a knapsack expression of depth at most two. Moreover, for $i \neq j, E_{i}$ and $E_{j}$ have no common variables. The existentially quantified variables $y_{1}, \ldots, y_{m}$ are the new variables that were introduced when splitting factors $u_{i}^{x_{i}}$ (e.g., $y_{2}, z_{2}$ in the formula $A_{1.1}$ ). The variables $z_{j}, z_{j}^{\prime}, z_{j}^{\prime \prime}$ in (3) are from $\left\{x_{1}, \ldots, x_{k}, y_{1}, \ldots, y_{m}\right\}$. The equations $z_{j}=z_{j}^{\prime}+z_{j}^{\prime \prime}+1$ in (3) result from the splitting of factors $u_{i}^{x_{i}}$. For instance, $x_{2}=y_{2}+1+z_{2}$ in $A_{1.1}$ is one such equation.

In order to bound the magnitude of sol $(E)$ it suffices to consider a single conjunctive formula of the form (3), since disjunction corresponds to union of semilinear sets, which does not increase the magnitude. We can also ignore the existential quantifiers in (3), because existential quantification corresponds to projection onto 
some of the coordinates, which cannot increase the magnitude. Hence, we have to consider the magnitude of the semilinear set $A$ defined by

$$
\bigwedge_{i \in I} E_{i}=1 \bigwedge_{j \in J} z_{j}=z_{j}^{\prime}+z_{j}^{\prime \prime}+1
$$

The splitting process that finally produces formula (4) can be seen as a tree $T$, where every node $v$ is labelled with a knapsack expression $E(v)$, the root is labelled with $E$, the leaves are labelled with the expressions $E_{i}(i \in I)$ from (3) and the children of a node $v$ are labelled with the expressions into which $E(v)$ is decomposed. The number of children of every node is at most three (three children are only produced in Case 2.6).

Let us first show that the size of this tree $T$ is bounded by $\mathcal{O}\left(k^{2}\right)$. We assign to each node $v$ of $T$ the number $d(v):=$ depth of the knapsack expression $E(v)$. Note that $d(v) \leq 2$ if and only if $v$ is a leaf. If $E(v)$ is split according to one of the Cases 2.1-2.6 then $v$ has $j \leq 3$ children $v_{1}, \ldots, v_{j}$, where $v_{1}, \ldots, v_{j-1}$ are leaves (their $d$-value is one or two) and $d\left(v_{j}\right)=d(v)-1$. If $E(v)$ is split according to Case 1.1 or 1.2 then $v$ has two children $v_{1}$ and $v_{2}$ such that (i) $d\left(v_{1}\right), d\left(v_{2}\right)<d(v)$, (ii) $d\left(v_{1}\right), d\left(v_{2}\right) \geq 2$ and $d\left(v_{1}\right)+d\left(v_{2}\right)=d(v)+1$ in Case 1.1, and (iii) $d\left(v_{1}\right), d\left(v_{2}\right) \geq 3$ and $d\left(v_{1}\right)+d\left(v_{2}\right)=d(v)+2$ in Case 1.2. Let $T^{\prime}$ be the tree that is obtained by removing all leaves with $d$-value at most 2 . It suffices to show that the size of $T^{\prime}$ is bounded by $\mathcal{O}\left(k^{2}\right)$. All leaves of $T^{\prime}$ have the $d$-value 3. Moreover, every non-leaf $v$ of $T^{\prime}$ has either exactly one child $v^{\prime}$ with $d(v)>d\left(v^{\prime}\right)$ or two children $v_{1}$ and $v_{2}$ such that $d(v) \geq d\left(v_{1}\right)+d\left(v_{2}\right)-2$. Let $n_{0}$ be the number of leaves of $T^{\prime}$ and $n_{2}$ be the number of nodes of $T^{\prime}$ with exactly two children. From the above equations, it follows that the root $r$ of $T^{\prime}$ satisfies $k=d(r) \geq 3 n_{0}-2 n_{2}$. Moreover, $n_{2}=n_{0}-1$. We get $k \geq n_{0}+2$, i.e., $n_{0} \leq k-2$ and $n_{2} \leq k-3$. Since every path from the root to a leaf can contain at most $k$ nodes having a single child, we must have $n_{1} \leq(k-2) k$. This shows that the size of $T^{\prime}$ and hence of $T$ is bounded by $\mathcal{O}\left(k^{2}\right)$. Thus, we also have $|I| \leq \mathcal{O}\left(k^{2}\right)$ in (4).

Next, we show that for every $i \in I,\left|E_{i}\right|$ is bounded polynomially in $|E|$. To see this, consider a single splitting step. In each of the above Cases 1.1-2.6 the argument is similar. Consider for instance Case 2.6, where the knapsack expression $E$ is replaced by three knapsack expressions $F_{t}, G_{t}, H_{t}$. We can bound the sizes of these expressions by $\left|F_{t}\right| \leq|E|+\left|u_{1,2}\right|+\left|u_{2,1}\right|+\left|w_{1}\right| \leq|E|+\left|u_{1}\right|+\left|u_{2}\right|+h$, $\left|G_{t}\right| \leq|E|+\left|u_{2,2}\right|+\left|u_{3,1}\right|+\left|w_{2}\right| \leq|E|+\left|u_{2}\right|+\left|u_{3}\right|+h+1$, and $H_{t}|\leq| E \mid+$ $\left|u_{1,1}\right|+\left|u_{3,2}\right|+|w| \leq|E|+\left|u_{1}\right|+\left|u_{3}\right|+2 h+1$. The number of splitting steps that finally leads to an $E_{i}$ is bounded by $k$ (since the depth of the knapsack expressions is reduced in each step). Hence, the size of each knapsack expression $E_{i}$ in (4) is bounded by $|E|+2 k|E|+k(2 h+1)=(2 k+1)|E|+k(2 \xi+2 \xi \log (2 k)+1) \leq \mathcal{O}\left(|E|^{2}\right)$. Since every $E_{i}$ has depth at most two, there is a fixed polynomial $p(n)$ such that the magnitude of every set $\operatorname{sol}\left(E_{i}\right)$ is bounded by $p(|E|)$. Hence, also $\bigoplus_{i \in I} \operatorname{sol}\left(E_{i}\right)$ is a semilinear set of magnitude at most $p(|E|)$ (the $\oplus$-operator on semilinear sets does not increase the magnitude). Note that $\bigoplus_{i \in I} \operatorname{sol}\left(E_{i}\right)$ is the semilinear set defined by the conjunction $\bigwedge_{i \in I} E_{i}=1$.

To bound the magnitude of the semilinear set $A$ defined by (4), one has to consider also the additional equations $z_{j}=z_{j}^{\prime}+z_{j}^{\prime \prime}+1$ for $j \in J$. Let $U$ be the set of variables that appear in the knapsack expressions $E_{i}(i \in I)$. Note that the dimension of $\bigoplus_{i \in I} \operatorname{sol}\left(E_{i}\right)$ is $|U|$. Since every knapsack expression $E_{i}(i \in I)$ contains at most two variables, we can bound the dimension of $\bigoplus_{i \in I} \operatorname{sol}\left(E_{i}\right)$ by $2|I| \leq \mathcal{O}\left(k^{2}\right)$. Note that for each equation $z_{j}=z_{j}^{\prime}+z_{j}^{\prime \prime}+1$ there exists a node $v$ in the tree $T$ with children $v^{\prime}, v^{\prime \prime}$ such that $z_{j}$ is a variable from $E(v), z_{j}^{\prime}$ is a variable from $E\left(v^{\prime}\right)$, and $z_{j}^{\prime \prime}$ is a variable from $E\left(v^{\prime \prime}\right)$. This implies that every variable $z_{j}$ 
is a sum of pairwise different variables from $U$ plus a constant that is bounded by $|T| \leq \mathcal{O}\left(k^{2}\right)$. Therefore the magnitude of $A$ is bounded by $\mathcal{O}\left(k^{2} \cdot p(|E|)\right)$, which is polynomial in $|E|$. This concludes the proof.

\section{More groups With KNAPSACK IN LogCFL}

Let $\mathcal{C}$ be the smallest class of groups such that (i) every hyperbolic group belongs to $\mathcal{C}$, (ii) if $G \in \mathcal{C}$ then also $G \times \mathbb{Z} \in \mathcal{C}$, and (iii) if $G, H \in \mathcal{C}$ then also $G * H \in \mathcal{C}$ (where $G * H$ is the free product of $G$ and $H$ ). The class $\mathcal{C}$ contains groups that are not hyperbolic (e.g., $\mathbb{Z} \times \mathbb{Z}$ ). From Theorem 8.1 and Proposition 5.1 we get:

Proposition 9.1. Every group from the class $\mathcal{C}$ is knapsack-tame and hence polynomially knapsack-bounded.

From Theorem 4.1 and 4.2 we get:

Proposition 9.2. Every group from the class $\mathcal{C}$ belongs to $O W$-AuxPDA.

Proposition 9.1 and 9.2 together with Theorem 6.1 and 6.2 yield:

Corollary 9.3. For every group $G$ from the class $\mathcal{C}$, membership for acyclic NFAs over $G$ and knapsack for $G$ both belong to LogCFL.

Corollary 9.3 generalizes Corollaries 7.2 and 7.3 as well as [4, Corollary 22], where it was shown that knapsack can be solved in polynomial time for a free product of hyperbolic groups and finitely generated abelian groups.

\section{Conclusion}

In this paper, it is shown that every hyperbolic group is knapsack-tame and that the knapsack problem can be solved in LogCFL. Here is a list of open problems that one might consider for future work.

- For the following important groups, it is not known whether the knapsack problem is decidable: braid groups $B_{n}$ (with $n \geq 3$ ), solvable BaumslagSolitar groups $\mathrm{BS}_{1, p}=\left\langle a, t \mid t^{-1} a t=a^{p}\right\rangle$ (with $p \geq 2$ ), and automatic groups which are not in any of the known classes with a decidable knapsack problem.

- In 13, it was shown that knapsack is decidable for every co-context-free group. The algorithm from [13] has an exponential running time. Is there a more efficient solution?

- Is there a polynomially knapsack-bounded group which is not knapsacktame?

\section{REFERENCES}

[1] Gerhard Buntrock and Friedrich Otto. Growing context-sensitive languages and ChurchRosser languages. Information and Computation, 141:1-36, 1998.

[2] Michael Elberfeld, Andreas Jakoby, and Till Tantau. Algorithmic meta theorems for circuit classes of constant and logarithmic depth. Electronic Colloquium on Computational Complexity (ECCC), 18:128, 2011.

[3] David B. A. Epstein and Derek F. Holt. The linearity of the conjugacy problem in wordhyperbolic groups. International Journal of Algebra and Computation, 16(2):287-306, 2006.

[4] Elizaveta Frenkel, Andrey Nikolaev, and Alexander Ushakov. Knapsack problems in products of groups. Journal of Symbolic Computation, 74:96-108, 2016.

[5] Moses Ganardi, Daniel König, Markus Lohrey, and Georg Zetzsche. Knapsack problems for wreath products. In Proceedings of STACS 2018, volume 96 of LIPICs, pages 32:1-32:13. Schloss Dagstuhl - Leibniz-Zentrum für Informatik, 2018.

[6] Etienne Ghys and Pierre de La Harpe. Sur les groupes hyperboliques d'après Mikhael Gromov. Progress in mathematics. Birkhäuser, 1990.

[7] Seymour Ginsburg and Edwin H. Spanier. Semigroups, Presburger formulas, and languages. Pacific Journal of Mathematics, 16(2):285-296, 1966. 
[8] Mikhail Gromov. Hyperbolic groups. In S. M. Gersten, editor, Essays in Group Theory, number 8 in MSRI Publ., pages 75-263. Springer, 1987.

[9] Christoph Haase. On the complexity of model checking counter automata. PhD thesis, University of Oxford, St Catherine's College, 2011.

[10] Derek F. Holt. Word-hyperbolic groups have real-time word problem. International Journal of Algebra and Computation, 10:221-228, 2000.

[11] Derek F. Holt, Markus Lohrey, and Saul Schleimer. Compressed decision problems in hyperbolic groups. In Proceedings of STACS 2019, volume 126 of LIPIcs, pages 37:1-37:16. Schloss Dagstuhl - Leibniz-Zentrum für Informatik, 2019.

[12] Richard M. Karp. Reducibility among combinatorial problems. In R. E. Miller and J. W. Thatcher, editors, Complexity of Computer Computations, pages 85-103. Plenum Press, 1972.

[13] Daniel König, Markus Lohrey, and Georg Zetzsche. Knapsack and subset sum problems in nilpotent, polycyclic, and co-context-free groups. In Algebra and Computer Science, volume 677 of Contemporary Mathematics, pages 138-153. American Mathematical Society, 2016.

[14] Eryk Kopczynski and Anthony Widjaja To. Parikh images of grammars: Complexity and applications. In Proceedings of LICS 2010, pages 80-89. IEEE Computer Society, 2010.

[15] Jörg Lehnert and Pascal Schweitzer. The co-word problem for the Higman-Thompson group is context-free. Bulletin of the London Mathematical Society, 39(2):235-241, 2007.

[16] Markus Lohrey. Decidability and complexity in automatic monoids. International Journal of Foundations of Computer Science, 16(4):707-722, 2005.

[17] Markus Lohrey and Georg Zetzsche. Knapsack in graph groups, HNN-extensions and amalgamated products. CoRR, abs/1509.05957, 2015.

[18] Markus Lohrey and Georg Zetzsche. Knapsack in graph groups, HNN-extensions and amalgamated products. In Proceedings of STACS 2016, volume 47 of LIPIcs, pages 50:1-50:14. Schloss Dagstuhl - Leibniz-Zentrum für Informatik, 2016.

[19] Markus Lohrey and Georg Zetzsche. Knapsack in graph groups. Theory of Computing Systems, 62(1):192-246, 2018.

[20] Alexei Mishchenko and Alexander Treier. Knapsack problem for nilpotent groups. Groups Complexity Cryptology, 9(1):87-98, 2017.

[21] Alexei Myasnikov and Andrey Nikolaev. Verbal subgroups of hyperbolic groups have infinite width. Journal of the London Mathematical Society, 90(2):573-591, 2014.

[22] Alexei Myasnikov, Andrey Nikolaev, and Alexander Ushakov. Knapsack problems in groups. Mathematics of Computation, 84:987-1016, 2015.

[23] Alexander Yu. Ol'shanskii. Almost every group is hyperbolic. International Journal of Algebra and Computation, 2(1):1-17, 1992.

[24] Ivan H. Sudborough. On the tape complexity of deterministic context-free languages. Journal of the ACM, 25(3):405-414, 1978.

[25] Anthony Widjaja To. Parikh images of regular languages: Complexity and applications. CoRR, abs/1002.1464, 2010. URL: http://arxiv.org/abs/1002.1464

[26] Heribert Vollmer. Introduction to Circuit Complexity. Springer-Verlag, 1999.

Universität Siegen, Germany

E-mail address: lohrey@eti.uni-siegen.de 